Article

\title{
Decoding of Mantle Processes in the Mersin Ophiolite, Turkey, of End-Member Arc Type: Location of the Boninite Magma Generation
}

\author{
Satoko Ishimaru ${ }^{1, *}$, Yuji Saikawa ${ }^{1}$, Makoto Miura ${ }^{2,3}$, Osman Parlak ${ }^{4,5}$ and Shoji Arai ${ }^{2}$ \\ 1 Department of Earth and Environmental Science, Faculty of Advanced Science and Technology, \\ Kumamoto University, 2-39-1 Kurokami, Chuo-ku, Kumamoto 860-8555, Japan; kcal93rakt@yahoo.co.jp \\ 2 Department of Earth Sciences, Kanazawa University, Kakuma, Kanazawa 920-1192, Japan; \\ makomiu1214@gmail.com (M.M.); ultrasa@staff.kanazawa-u.ac.jp (S.A.) \\ 3 GIA Godo Kaisha, 4-19-9 Taito, Taito-ku, Tokyo 110-0016, Japan \\ 4 Jeoloji Mühendisliği Bŏlümü, Çukurova Üniversitesi, TR-01330 Adana, Turkey; parlak@cu.edu.tr \\ 5 State Key Laboratory of Geological Processes and Mineral Resources, Center for Global Tectonics, \\ School of Earth Sciences, China University of Geosciences, Wuhan 430074, China \\ * Correspondence: s_ishimaru@kumamoto-u.ac.jp; Tel.: +81-96-342-3471
}

Received: 4 September 2018; Accepted: 15 October 2018; Published: 18 October 2018

\begin{abstract}
The Mersin ophiolite, Turkey, is of typical arc type based on geochemistry of crustal rocks without any signs of mid-ocean ridge (MOR) affinity. We examined its ultramafic rocks to reveal sub-arc mantle processes. Mantle peridotites, poor in clinopyroxene ( $<1.0 \mathrm{vol} . \%)$, show high Fo content of olivine (90-92) and $\mathrm{Cr} \#[=\mathrm{Cr} /(\mathrm{Cr}+\mathrm{Al})$ atomic ratio $](=0.62-0.77)$ of chromian spinel. $\mathrm{NiO}$ content of olivine is occasionally high (up to $0.5 \mathrm{wt} . \%$ ) in the harzburgite. Moho-transition zone (MTZ) dunite is also highly depleted, i.e., spinel is high Cr\# (0.78-0.89), clinopyroxene is poor in HREE, and olivine is high Fo (up to 92), but relatively low in $\mathrm{NiO}(0.1-0.4 \mathrm{wt} . \%)$. The harzburgite is residue after high-degree mantle melting, possibly assisted by slab-derived fluid. The high-Ni character of olivine suggests secondary metasomatic formation of olivine-replacing orthopyroxene although replacement textures are unclear. The MTZ dunite is of replacive origin, resulted from interaction between $\mathrm{Mg}$-rich melt released from harzburgite diapir and another harzburgite at the diapir roof. The MTZ dunite is the very place that produced the boninitic and replacive dunite. The MTZ is thicker $(>1 \mathrm{~km})$ in Mersin than in MOR-related ophiolite (mostly $<500 \mathrm{~m}$ ), and this is one of the features of arc-type ophiolite.
\end{abstract}

Keywords: arc-type ophiolite; mantle wedge; partial melting; metasomatism; peridotite-melt reaction; boninite; Mersin; Turkey

\section{Introduction}

The mantle wedge is the very place where both magmatism and metasomatism are active, and is considered to be complicated in petrologic nature (e.g., [1]). It is quite important to accumulate petrologic data for our better understanding of the mantle wedge. Mantle peridotite xenoliths from current island arcs have been a good source of information about the mantle wedge because of the absence of serpentinization, but their small sizes ( $<1 \mathrm{~m}$ across) and fragmentary nature lead to a serious disadvantage in terms of spatial resolution of mantle processes. The mantle section of ophiolites of arc origin may provide us with information to delineate details of the mantle wedge processes because of its greater exposure. For example, the Oman ophiolite has a mantle section of $\sim 500 \mathrm{~km}$ in length [2]. However, ophiolites have various origins (e.g., [3]), and therefore, we should carefully select ophiolites for the purpose of investigation of the mantle wedge process. Typical ophiolites including the Tethyan 
ones experienced switch of tectonic setting of formation from a mid-ocean ridge to an island arc [4-6]. They may exhibit various degrees of superposition of island-arc rocks on precursor oceanic rocks [4,7]. We selected the Mersin ophiolite, one of Neothetyan ophiolites in Turkey (e.g., [8]) for our study because its crustal rocks, except for alkaline basalt showing pre-ophiolite feature [9], mostly show island-arc characters without any sign of MORB affinity $[9,10]$. We expect that the mantle section of the Mersin ophiolite has recorded various mantle-wedge processes related to production of the arc-type crustal rocks upsection.

\section{Geological Setting}

\subsection{Regional Geology}

Anatolia (Asia Minor) is subdivided into EW-trending belts from North to South, namely the Pontides, the Anatolides, the Taurides and Southeast Anatolian Border Folds [11] (Figure 1a). Ophiolites and related metamorphic rocks in Turkey define several suture zones that resulted from the closure of the Neotethyan oceanic basins between the Eurasian and Afro-Arabian plates during the Triassic to Miocene period. From North to South, these suture zones are named: (a) The Intra-Pontide, (b) the İzmir-Ankara-Erzincan; (c) the Inner Tauride; and (d) the SE Anatolian suture zones [12-25].
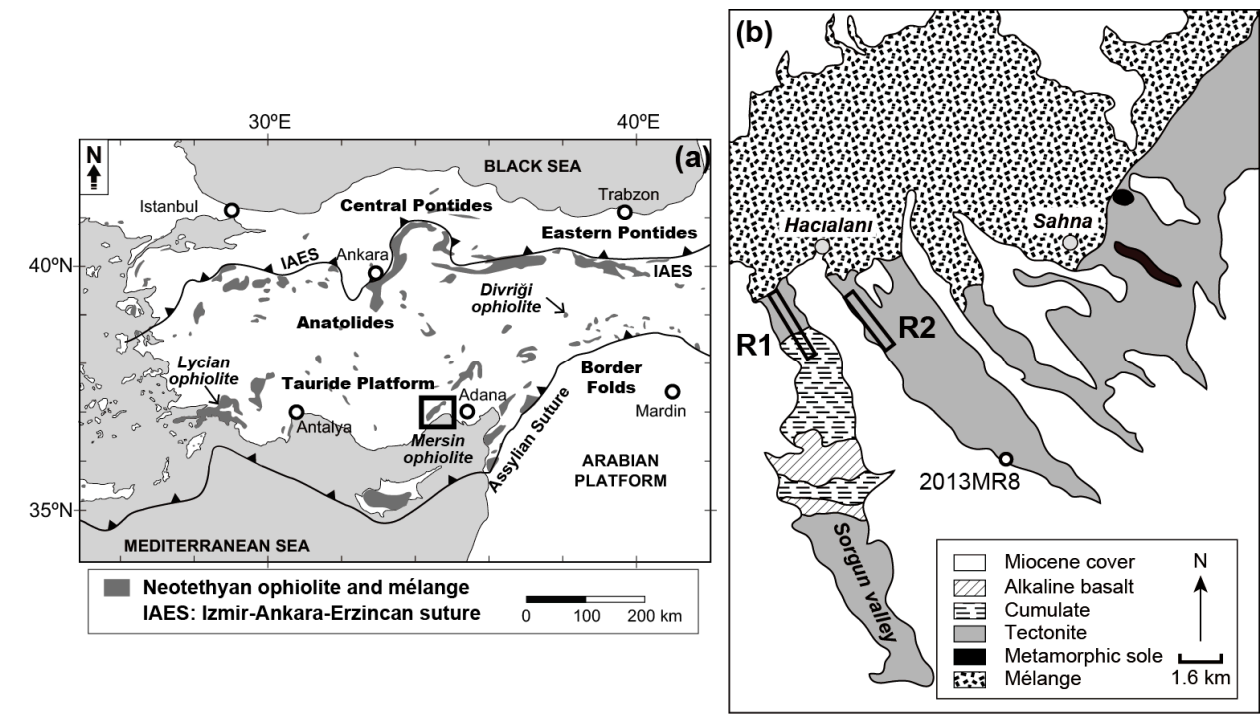

Figure 1. (a) Distributions of Neotethyan ophiolites and mélanges in Turkey. Modified after Parlak [8]. (b) Simplified geological map of the Mersin ophiolite [8]. R1 and R2 are routes for taking samples for this study and the details are in Figure 2.

The ophiolites of Southern Turkey are located along two lineaments, namely the Assylian suture zone and the Tauride belt (Figure 1a). The Tauride ophiolites are characterized by dismembered ophiolitic units, namely the Lycian, Tekirova, Beysehir-Hoyran, Alihoca, Mersin, Pozanti-Karsanti, Pinarbasi, and Divrigi ophiolites [8,12,19,26-28]. The Tauride ophiolites mainly consist of three tectonic units, they are, in ascending order, ophiolitic mélange, sub-ophiolitic metamorphic sole, and oceanic lithospheric remnants [8].

The Mersin ophiolite is situated in the Southern flank of the Tauride platform and located approximately $30 \mathrm{~km}$ NW of Mersin town in Southern Turkey (Figure 1a). It is exposed in an area of $60 \mathrm{~km}$ long-25 km wide and displays an approximately $6 \mathrm{~km}$-thick crust-mantle section (Figure 1b). The Mersin ophiolite shows a good ophiolitic sequence except for the absence of sheeted dykes, and the stratigraphy is as follows from bottom to top: Mantle tectonites with an underlying metamorphic sole, ultramafic-mafic cumulates, and effusive rocks [29]. The mantle tectonites are highly serpentinized along the contact with sole amphibolite or the Mersin mélange, and exhibit foliation characterized 
by alteration of dunite and orthopyroxenite bands [29]. We observed a dunite-dominant zone with chromitite layering and pods between tectonized peridotite and ultramafic-mafic cumulative rocks showing layering (Figure 2) [30]. We define this dunite-dominant zone as the Moho-transition zone, which is mainly composed of dunite with minor amount of wehrlite [31]. This Moho-transition zone dunite changes to ultramafic cumulates, represented by dunite, wehrlite, clinopyroxenite, and gabbro upsection [30]. The frequency of wehrlite increases upward within the ultramafic cumulate zone, which is approximately $1 \mathrm{~km}$ thick [8]. The mafic cumulates, approximately $2 \mathrm{~km}$ thick, shows rhythmic to graded layering, and is dominated by olivine gabbro, leucogabbro, gabbro, and anorthosites [30]. There are two types of effusive rocks, i.e., alkaline and tholeiitic basalts, in the Mersin ophiolite. The former with seamount-type alkaline geochemical character having interlayers of radiolarian cherts and pelagic limestones yielded Late Jurassic-Early Cretaceous age, while the latter with island arc tholeiite (IAT) geochemical character yielded Late Cretaceous age [9]. The ophiolite-related metamorphic rocks, i.e., metamorphic sole, are observed at the base of the thick mantle tectonite, and the Mersin mélange is underneath. The details about the sub-ophiolitic sequence, i.e., metamorphic sole and mélange, are in Parlak and Robertson [27] and Parlak et al. [32]. A number of dykes of dolerite and microgabbro cut the entire sequences, even in metamorphic soles, although not cut the Mersin mélange and the platform carbonates [8,33]. The dykes show IAT-like features [10] and the timing of their intrusion is interpreted as post-metamorphism based on the absence of ductile deformation and metamorphic texture [32].

Age of the Mersin ophiolite has been estimated by using whole-rock ${ }^{40} \mathrm{Ar} /{ }^{39} \mathrm{Ar}$ and $\mathrm{K}-\mathrm{Ar}$ techniques for isolated dykes cutting metamorphic sole and mantle teconite, and ranges from 91 to $86 \mathrm{Ma}[10,34]$. In addition, ${ }^{40} \mathrm{Ar} /{ }^{39} \mathrm{Ar}$ ages of $96-90 \mathrm{Ma}$ for amphibolites (or hornblende separates of those) were also presented [10,34].

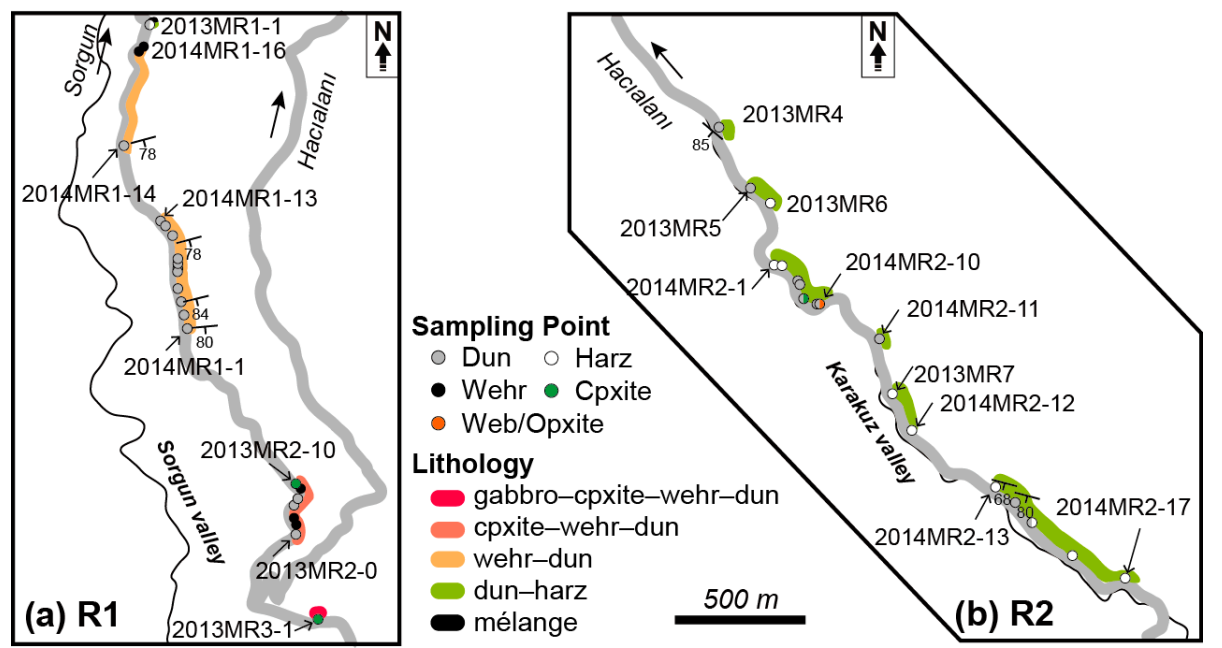

Figure 2. Lithological maps along the routes. (a) Route 1 (R1). (b) Route 2 (R2). Circles show the lithology of collected samples. Dun, dunite; Harz, harzburgite; Wehr, wehrlite; Cpxite, clinopyroxenite; Web, websterite; and Opxite, orthopyroxenite. More detailed information is shown in Table 1.

\subsection{Sampling}

In the Mersin ophiolite, the upper part of the ophiolitic sequence (effusive rocks, gabbros, ultramafic, and mafic cumulates) is available in the Westernmost area (Figure 1b), while the lower part (mantle tectonite) with the underlying metamorphic sole, is exposed mainly in the North and Eastern regions (Figure 1b). We collected samples from the Western end of the Mersin ophiolite along the Sorgun valley (Route 1: R1) and Karakuz valley next to the Sorgun valley (Route 2: R2) (Figures 1b and 2; Table 1). We call ultramafic lithologies from the upper section downward as follows: Layered ultramafic (LU) rocks for the cumulates of Parlak et al. [9], Moho-transition zone (MTZ) peridotites for 
the mantle tectonite of Parlak et al. [9] along the Sorgun valley (R1), and mantle peridotites for the mantle tectonite of Parlak et al. [9] along the Karakuz valley, respectively (cf. Figure 1b). Along the Sorgun valley, R1, we observed good exposure of MTZ peridotite with minor amount of ultramafic and mafic-layered rocks (LU rocks) (Figures $1 \mathrm{~b}$ and 2; [30]) and the total thickness of the layered rocks is over $3 \mathrm{~km}$ [30]. The Mersin mélange is underneath the ophiolite, and there is no metamorphic sole in this region (Figures $1 \mathrm{~b}$ and $2 ;[30]$ ). The detailed petrological and geochemical descriptions of the mafic-ultramafic-layered rocks are in [29,30]. Along the Karakuz valley, R2, we observed mantle peridotites (mainly harzburgite and dunite), although it is not clear stratigraphically how lower these mantle peridotites are than the base of the MTZ peridotites of R1 [29,30] (Figure 1b).

We started sampling from the clinopyroxenite of the LU rocks (upper) to harzburgite of the MTZ peridotites (lower) in R1 (Figures 1b, 2 and 3; Table 1). Clinopyroxenite and wehrlite of the LU rocks show nice layering (Figure 4a). Most of the MTZ peridotites are dunite with a minor amount of harzburgite and wehrlite. Harzburgite occurs only just above the Mersin mélange and coexists with dunite in R1. However, we could not observe their relationships on the outcrop because of high degrees of shearing and serpentinization (Figures $2 \mathrm{a}$ and 3). Chromite layers were observed in both the LU rocks and the MTZ dunite (Figure $4 \mathrm{~b}$ ), and their strikes and dips are about N82 ${ }^{\circ} \mathrm{E} 84^{\circ} \mathrm{S}$ in the $\mathrm{LU}$ rocks and $\mathrm{N} 80^{\circ} \mathrm{E} 78^{\circ} \mathrm{S}$ in the MTZ rocks (Figure 2a; Table 1). There is a thick dolerite dike in dunite of MTZ peridotites, and the strike is discordant to the chromitite layer.

(a) R1

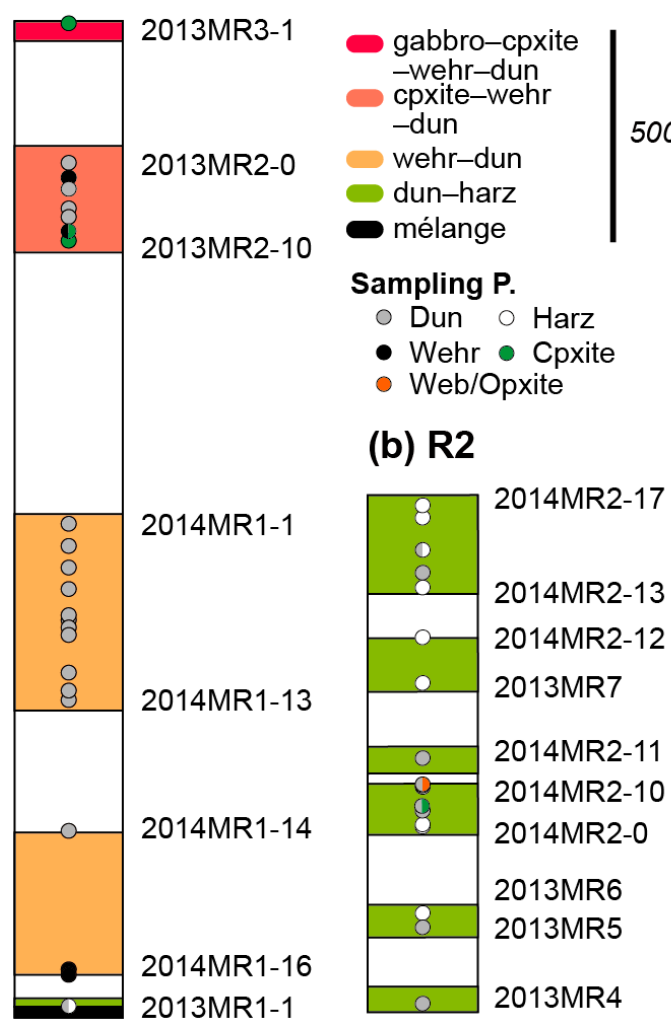

Figure 3. Simplified stratigraphic column. Circles, "Sampling P. (points)", show the lithology of collected samples. (a) R1. (b) R2. Symbols are the same as those in Figure 2. More detailed information is shown in Table 1. Abbreviations are the same as Figure 2. 
Table 1. Outcrop and sample descriptions. Samples are listed from top to bottom based on the stratigraphic column (Figure 3). Sampling points without any information of location are paced by feet and plotted in Figures 2 and 3.

\begin{tabular}{|c|c|c|c|c|c|c|}
\hline Route & Sample & Rock Type & Latitude & Longitude & Foliation & Notes \\
\hline R1 & \multicolumn{6}{|c|}{ Layered Ultramafic (LU) rocks } \\
\hline & 2013MR3-01 & Cpxite & $\mathrm{N} 36^{\circ} 48.551^{\prime}$ & $\mathrm{E} 34^{\circ} 10.350^{\prime}$ & & Gabbro-cpxite \\
\hline & 2013MR3-02 & Wehrlite & & & & Cpxite in wehrlite \\
\hline & 2013MR2-0 & Dunite & & & & \\
\hline & 2013MR2-02 & Wehrlite & & & & \\
\hline & 2013MR2-06 & Wehrlite & & & & \\
\hline & 2013MR2-07 & Dunite & & & & \\
\hline & 2013MR2-08 & Cpxite & & & & \\
\hline & 2013MR2-09 & Wehrlite & & & & \\
\hline & 2013MR2-10 & Cpxite & & & & \\
\hline & \multicolumn{6}{|c|}{ Moho transition zone (MTZ) rocks } \\
\hline & 2014MR1-01 & Dunite & $\mathrm{N} 36^{\circ} 49.214^{\prime}$ & $\mathrm{E} 34^{\circ} 09.938^{\prime}$ & $\mathrm{EW} 80^{\circ} \mathrm{S}$ & \\
\hline & 2014MR1-03 & Dunite & $\mathrm{N} 36^{\circ} 49.250^{\prime}$ & E $34^{\circ} 09.954^{\prime}$ & $\mathrm{N} 82^{\circ} \mathrm{E} 84^{\circ} \mathrm{S}$ & \\
\hline & 14T-MR01 & Chromitite & & & & Massive chromitite \\
\hline & 14T-MR03 & Dunite & & & & Host dunite of 14T-MR01 \\
\hline & 2014MR1-04 & Dunite & $\mathrm{N} 36^{\circ} 49.289^{\prime}$ & $\mathrm{E} 34^{\circ} 09.933^{\prime}$ & & \\
\hline & 2014MR1-05 & Dunite & $\mathrm{N} 36^{\circ} 49.315^{\prime}$ & $\mathrm{E} 34^{\circ} 09.925^{\prime}$ & & \\
\hline & 2014MR1-06 & Dunite & $\mathrm{N} 36^{\circ} 49.358^{\prime}$ & $\mathrm{E} 34^{\circ} 09.918^{\prime}$ & & \\
\hline & 2014MR1-09 & Dunite & $\mathrm{N} 36^{\circ} 49^{\prime} 433^{\prime}$ & $\mathrm{E} 34^{\circ} 09^{\prime} 910^{\prime}$ & $\mathrm{N} 80^{\circ} \mathrm{E} 78^{\circ} \mathrm{SE}$ & \\
\hline & 2014MR1-13 & Dunite & & & & \\
\hline & 2014MR1-14 & Dunite & $\mathrm{N} 36^{\circ} 49.714^{\prime}$ & $\mathrm{E} 34^{\circ} 09.724^{\prime}$ & $\mathrm{N} 80^{\circ} \mathrm{E} 78^{\circ} \mathrm{SE}$ & \\
\hline & 2014MR1-16 & Wehrlite & & & & \\
\hline & 2013MR1-02 & Harz & $\mathrm{N} 36^{\circ} 50.081^{\prime}$ & $\mathrm{E} 34^{\circ} 09.787^{\prime}$ & & \\
\hline \multirow[t]{24}{*}{$\mathrm{R} 2$} & Mantle rocks & & & & & \\
\hline & 2014MR2-16 & Harz & $\mathrm{N} 36^{\circ} 48.535^{\prime}$ & $\mathrm{E} 34^{\circ} 13.229^{\prime}$ & & \\
\hline & 2014MR2-15 & Harz & $\mathrm{N} 36^{\circ} 48.608^{\prime}$ & $\mathrm{E} 34^{\circ} 13.114^{\prime}$ & & \\
\hline & 2014MR2-14 & Dunite & $\mathrm{N} 36^{\circ} 48.653^{\prime}$ & $\mathrm{E} 34^{\circ} 13.067^{\prime}$ & $\mathrm{N} 70^{\circ} \mathrm{W} 80^{\circ} \mathrm{SW}$ & \\
\hline & 2014MR2-13 & Harz & $\mathrm{N} 36^{\circ} 48.688^{\prime}$ & $\mathrm{E} 34^{\circ} 13.010^{\prime}$ & $\mathrm{N} 70^{\circ} \mathrm{W} 68^{\circ} \mathrm{SW}$ & \\
\hline & 2014MR2-12 & Harz & $\mathrm{N} 36^{\circ} 48.809^{\prime}$ & $\mathrm{E} 34^{\circ} 12.751^{\prime}$ & & \\
\hline & 2013MR7-01 & Harz & $\mathrm{N} 36^{\circ} 48.901^{\prime}$ & $\mathrm{E} 34^{\circ} 12.702^{\prime}$ & & \\
\hline & 2014MR2-11 & Dunite & $\mathrm{N} 36^{\circ} 49.026^{\prime}$ & $\mathrm{E} 34^{\circ} 12.674^{\prime}$ & & \\
\hline & 2014MR2-10 & Websterite & $\mathrm{N} 36^{\circ} 49.117^{\prime}$ & $\mathrm{E} 34^{\circ} 12.490^{\prime}$ & & Websterite dike in dunite \\
\hline & 2014MR2-09 & Dunite/Harz & $\mathrm{N} 36^{\circ} 49.113^{\prime}$ & $\mathrm{E} 34^{\circ} 12.480^{\prime}$ & & Dunite-Harz boundary \\
\hline & 2014MR2-07 & Dunite & $\mathrm{N} 36^{\circ} 49.126^{\prime}$ & E $34^{\circ} 12.442^{\prime}$ & & Dunite with websterite dike \\
\hline & 2014MR2-06 & Harz & & & & \\
\hline & 2014MR2-05 & Dunite & $\mathrm{N} 36^{\circ} 49.159^{\prime}$ & $\mathrm{E} 34^{\circ} 12.430^{\prime}$ & & Host dunite of 14T-MR12 \\
\hline & 14T-MR12 & Chromitite & & & & Banded chromitite \\
\hline & 2014MR2-03 & Harz & $\mathrm{N} 36^{\circ} 49.199^{\prime}$ & $\mathrm{E} 34^{\circ} 12.374^{\prime}$ & \multirow{4}{*}{\multicolumn{2}{|c|}{$\mathrm{N} 30^{\circ} \mathrm{W} 78^{\circ} \mathrm{W}$}} \\
\hline & 14T-MR04 & Wehrlite & $\mathrm{N} 36^{\circ} 49.202^{\prime}$ & $\mathrm{E} 34^{\circ} 12.381^{\prime}$ & & \\
\hline & 2014MR2-01 & Harz & $\mathrm{N} 36^{\circ} 49.205^{\prime}$ & $\mathrm{E} 34^{\circ} 12.346^{\prime}$ & & \\
\hline & 2013MR5-01 & Dunite & $\mathrm{N} 36^{\circ} 49.394^{\prime}$ & $\mathrm{E} 34^{\circ} 12.267^{\prime}$ & & \\
\hline & 2013MR5-02 & Websterite & & & \multirow{5}{*}{$\mathrm{N} 45^{\circ} \mathrm{W} 85^{\circ} \mathrm{SW}$} & Websterite dike in dunite \\
\hline & 2013MR4-02 & Opxite & $\mathrm{N} 36^{\circ} 49.557$ & $\mathrm{E} 34^{\circ} 12.158^{\prime}$ & & Harz with opxite dike \\
\hline & 2013MR4-03 & Dunite & & & & \multirow{4}{*}{ Dunite with websterite dike } \\
\hline & 2013MR4-04 & Harz & & & & \\
\hline & 2013MR4-05 & Dunite & & & & \\
\hline & 2013MR8-01 & Harz & $\mathrm{N} 36^{\circ} 45.789^{\prime}$ & $\mathrm{E} 34^{\circ} 16.019^{\prime}$ & $\begin{array}{c}\mathrm{N} 70^{\circ} \mathrm{W} \\
64^{\circ} \mathrm{S}\end{array}$ & \\
\hline
\end{tabular}

Abbreviations of rock lithology are the same as Figure 2. 


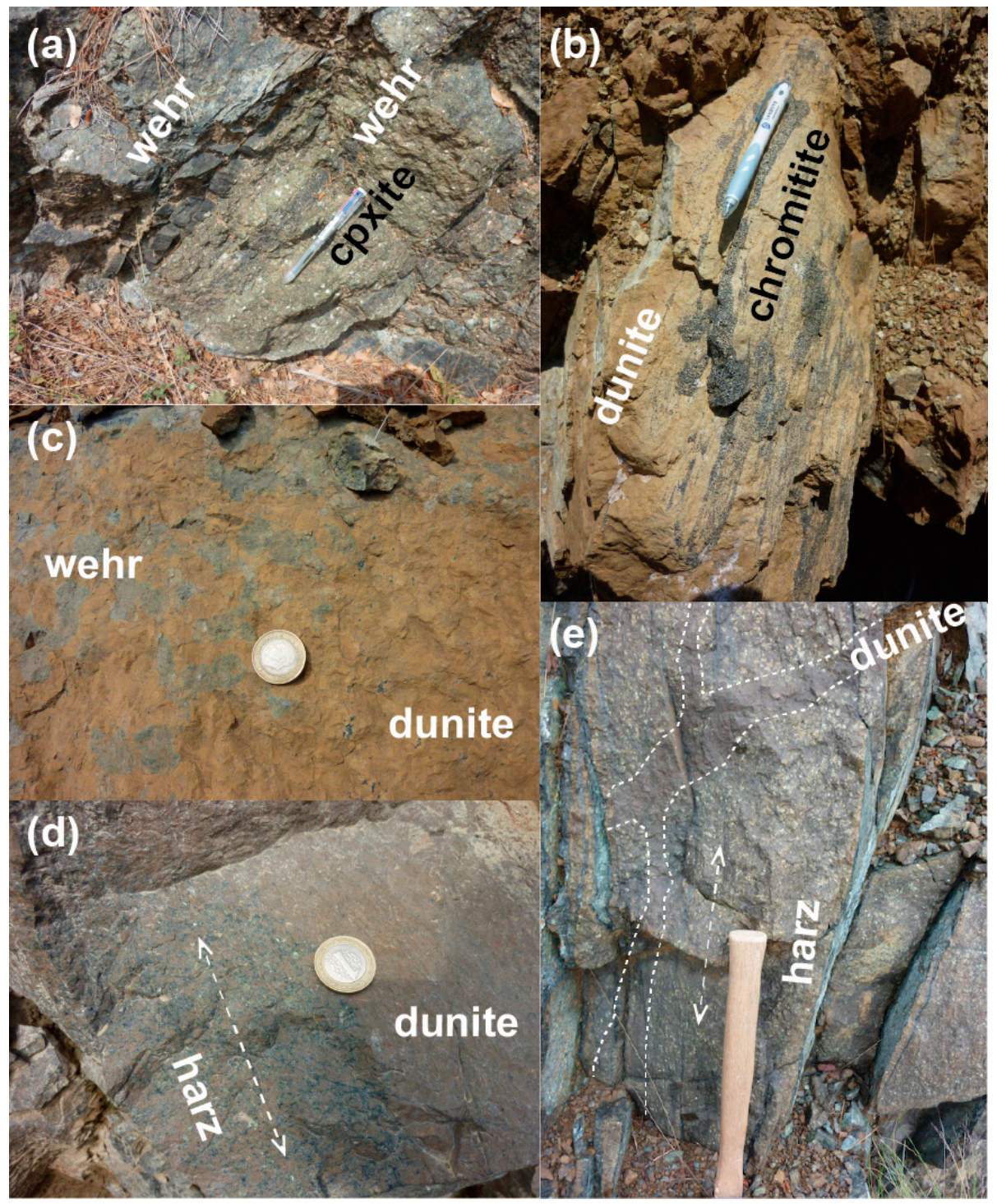

Figure 4. Photographs of representative lithology on outcrop. (a) Layering of clinopyroxenite (2013MR2-08) and wehrlite of LU (2013MR2-09). (b) Banded chromitite in LU dunite of R1(2014MR1-03). (c) Wehrlitic part containing relatively coarse clinopyroxenes in mantle dunite in R2 (2014MR2-03). (d) Boundary between dunite and harzburgite of mantle rocks in R2 (2014MR2-04). A double-headed arrow roughly shows foliation direction of harzburgite. (e) Discordant dunite band (smooth brownish part and enclosed by white dotted lines) in mantle harzburgite in R2 (2014MR2-17). A double-headed arrow roughly shows foliation direction of harzburgite.

In R2, we collected harzburgite and dunite of mantle peridotite with minor amount of pyroxenites, i.e., clinopyroxenite, orthopyroxenite and orthopyroxene-rich olivine websterite (Figures 2 and 3). The pyroxenites are mainly observed as discordant dike, cross-cutting the harzburgite foliation. The Northern end of the R2 is interpreted as a stratigraphically lower section of mantle peridotites based on field observation of strikes and dips of harzburgite foliation (Figures $2 b$ and $3 b$; Table 1 ). Some dunites have coarse clinopyroxene patches and/or seams (Figure 4c), and are discordant to the harzburgite foliation (Figure $4 \mathrm{~d}$,e). Orthopyroxene gradually decreases in mode from harzburgite to dunite at their boundary. Banded chromitite was observed also in the R2 mantle dunite. 


\section{Petrographic Features of Ultramafic Rocks}

The samples are highly serpentinized (up to $96 \mathrm{vol} \%$ ) and the degree of serpentinization is lower in wehrlite (60 80 vol.\%) and harzburgite (50 87 vol.\%) than in dunite (71 96 vol.\%). We determined their modal compositions using point counter ( 2000 counts in total) and the primary lithology was determined all serpentine is the secondary phase after olivine except for pseudomorphs of pyroxenes (Figure 5). If there is pyroxene pseudomorph with orthogonal and oblique extinction, we accounted for the pseudomorph as orthopyroxene and clinopyroxene, respectively.

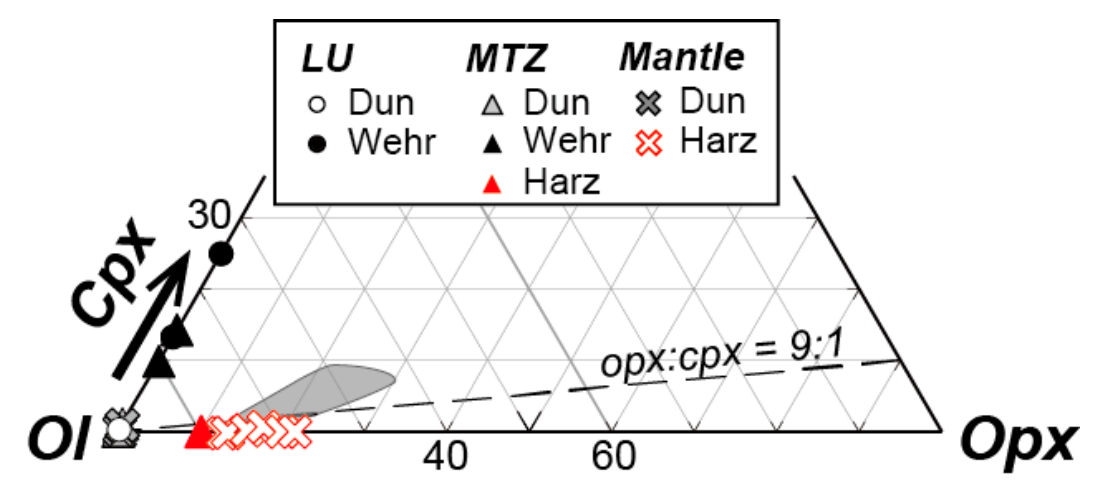

Figure 5. Estimated modal compositions of the Mersin ultramafic rocks. We assumed all serpentine as secondary phase after olivine unless obvious feature of pseudomorphs after pyroxenes, e.g., cleavage, was recognized (see text). Dashed line represents clinopyroxene (cpx)/orthopytoxene (opx) = 1:9 ratio, indicating the possible presence of clinopyroxene at solidus temperatures above this line [35].

\subsection{LU Rocks (Dunite-Wehrlite-Clinopyroxenite of R1)}

Calculated modal composition of dunite is $\approx 98$ vol. $\%$ olivine, $0.8 \sim 1.4$ vol. $\%$ chromian spinel and $<2.6$ vol.\% clinopyroxene (Figure 5). All dunites are almost mono-mineralic but clinopyroxene rarely occurs as an interstitial phase between olivine grains. Chromian spinel shows euhedral to subhedral shape and rarely contains clinopyroxene inclusions.

Calculated modal composition of wehrlite is $75.0 \sim 86.3$ vol. $\%$ olivine, $<0.2$ vol. $\%$ chromian spinel and 13.4 25.0 vol.\% clinopyroxene (Figure 5). Clinopyroxene is coarser (up to $1 \mathrm{~cm}$ ) in wehrlite than in dunite, and is usually anhedral to subhedral but occasionally occurs as an interstitial phase to olivine grains (Figure 6a). Chromian spinel is euhedral to subhedral and usually fine $(<100 \mu \mathrm{m})$ (Figure 6a).

Clinopyroxenite shows lower degrees of serpentinization ( $<8.0 \mathrm{vol} . \%)$ and modal composition of clinopyroxenite is $4.5 \sim 9.0$ vol. $\%$ olivine, $<0.2$ vol. $\%$ chromian spinel, $0.2 \sim 0.7$ vol. $\%$ orthopyroxene and 90.7 95.2 vol.\% clinopyroxene. Grain size of minerals is usually large (up to $1 \mathrm{~cm}$ ), but chromian spinel and olivine is usually fine. Chromian spinel grains show anhedral to subhedral shapes. Orthopyroxene is subhedral to anhedral, and frequently altered and occurs as pseudomorph. 


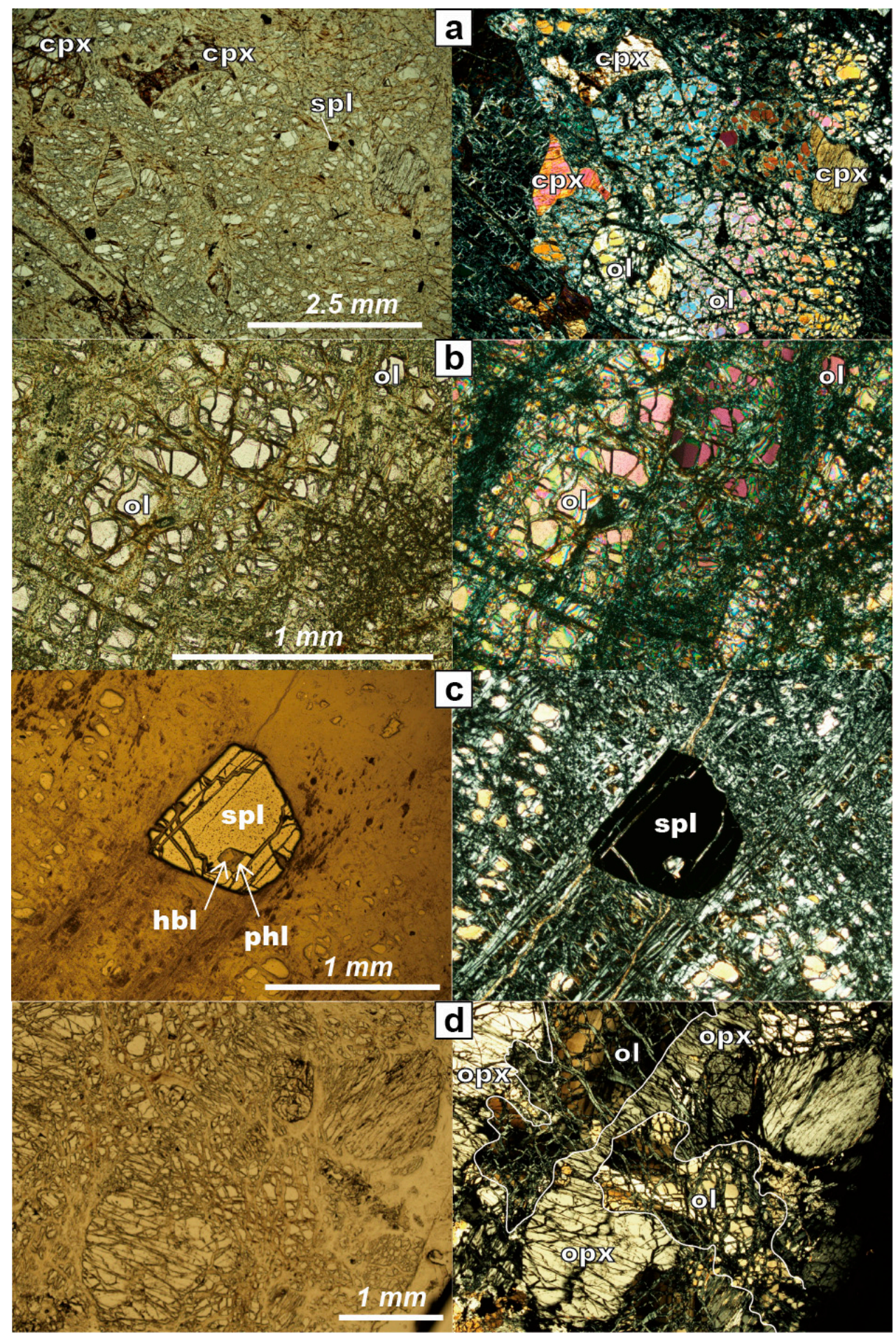

Figure 6. Photomicrographs of representative lithologies of the Mersin ultramafic rocks. Left and right panels are plane-polarized and crossed-polarized images, respectively, except for the left image of panel (c), which is reflected-light image. Abbreviations are as follows: cpx, clinopyroxene; ol, olivine; spl, chromian spinel; hbl, hornblende; phl, phlogopite; and opx, orthopyroxene. (a) LU wehrlite (2013MR2-02). (b) MTZ dunite (2014MR1-09). Olivine at the center of the photo show kink bands. (c) Inclusion of hornblende + phlogopite in chromian spinel in the mantle dunite (2014MR2-11). (d) Orthopyroxene consuming coarse olivine grain in an orthopyroxene-rich part of the mantle harzburgite (2014MR2-13). 


\subsection{MTZ Peridotites (Dunite-Wehrlite-Harzburgite of R1)}

All types of lithology in this group show higher degree of serpentinization than equivalents of the LU rocks (Figure 6a,b).

Mineral assemblage of MTZ dunite is the same as that of the LU rocks and the degree of serpentinization is higher (89.6 95.5 vol.\%) than in the LU rocks. The relationship between olivine and clinopyroxene is, however, unclear due to high degree of serpentinization. Modal amount of olivine is quite high (>97 vol.\%), and chromian spinel is higher in mode (0.9 2.5 vol.\%) than in the LU rocks. Some olivine grains show wavy extinction and kink banding (Figure 6b). Modal amount of clinopyroxene ( $<1.0 \mathrm{vol} . \%$ ) is lower than in the LU rocks, and the size is also small (up to $500 \mu \mathrm{m}$ ). Chromian spinel is euhedral to subhedral and rarely contains inclusions.

Massive chromitite (14T-MR01) is mainly composed of chromian spinel with minor amount of chlorite and serpentine. Olivine is completely serpentinized or altered. Chlorite occurs solely around chromian spinel or inside as inclusions. Amphiboles also occasionally occur as inclusion in chromian spinel.

We found wehrlite at a few outcrops in MTZ (Table 1), and its mineral assemblage is the same as that in the LU rocks. Modal amount is varied (9.9 14.2 vol.\%) for clinopyroxene and almost the constant (0.4 0.9 vol.\%) for chromian spinel. Grain size of clinopyroxene is almost the same as that in the LU rocks and the shape is subhedral. Chromian spinel grains show euhedral to subhedral shapes.

MTZ harzburgite is also highly serpentinized (79.6 vol.\%), and calculated modal amounts of olivine, orthopyroxene, chromian spinel are 89.7 vol.\%, 10.1 vol.\%, and 0.2 vol.\%, respectively. Clinopyroxene is fine in size $(<100 \mu \mathrm{m})$ and very small in amount. Orthopyroxene, partly to completely altered, show two types: One shows rounded shapes and the other showing subhedral shape, of which rim is interstitial to olivine grains. The orthopyroxene is usually coarse (up to $1 \mathrm{~mm}$ ) and there is no obvious relationship between the shape and the size. Chromian spinel is opaque and shows subhedral to anhedral shapes in thin section.

\subsection{Mantle Peridotites (Ultramafic Rocks of R2)}

Mantle dunite in R2 is less serpentinized (71.4 88.7 vol.\%) than that in R1, but the modal amounts of clinopyroxene and chromian spinel are not so different between the two $(0.3 \sim 0.9$ vol.\% and 1.3 2.4 vol.\%, respectively). The size of clinopyroxene is far smaller $(\approx 100 \mu \mathrm{m})$ than in dunites from the LU rocks and MTZ, but rarely large $(\approx 1 \mathrm{~mm})$. Olivine is usually coarse (up to $5 \mathrm{~mm}$ ), and shows weak wavy extinction. Chromian spinel grains show euhedral to subhedral rounded shapes, and rarely contains inclusions of hornblende + phlogopite \pm clinopyroxene (Figure $6 c$ ).

Clinopyroxenite in this mantle peridotite zone is mainly composed of clinopyroxene (91.6 93.1 vol.\%) with appreciable amounts of olivine ( $<8.0 \mathrm{vol} . \%)$ and orthopyroxene $(\approx 0.5 \mathrm{vol} . \%)$ and a trace amount of chromian spinel ( $<0.2 \mathrm{vol} . \%)$. The degree of serpentinization is quite low $(<10 \mathrm{vol} . \%)$ relative to other lithologies in all others.

Orthopyroxene-rich olivine websterite and olivine orthopyroxenite is composed of orthopyroxene, clinopyroxene and olivine with a minor amount of chromian spinel. Orthopyroxene is very coarse (up to $2 \mathrm{~cm})$ and highly kinked but partly altered. Olivine and clinopyroxene are basically small $(\sim 500 \mu \mathrm{m})$. Amphiboles occur as lamellar inclusions after clinopyroxene lamellae in orthopyroxene, as well as discrete grains forming veinlets. Chromian spinel is subhedral and rounded in shape.

The mantle harzburgite is the same in mineral assemblage as that of MTZ harzburgite but is lower in the degree of serpentinization (50.6 79.2 vol.\%) than the MTZ harzburgite. There is no obvious relationship between the degree of serpentinization or modal amounts of pyroxenes and the stratigraphical position (=depth from the MTZ); modal amounts of orthopyroxene and clinopyroxene vary from 12.6 to 21.5 vol.\% and from a trace to 0.7 vol.\%, respectively (Figure 5). Some of olivine and orthopyroxene grains show wavy extinction and kink banding (Figure $6 \mathrm{~d}$ ). The orthopyroxene is the same in shape as that in MTZ harzburgite, and it is noteworthy that some orthopyroxene 
grains appears to consuming coarse olivine grains (Figure 6d). Chromian spinel shows subhedral to anhedral shapes.

Banded chromitites show graded bedding, and olivine and clinopyroxene are in part preserved in a spinel-poor zone. In this case, chlorite is absent around chromian spinel, although present as inclusions in spinel. Olivine and clinopyroxene also occur as inclusion in chromian spinel, and clinopyroxene rarely coexists with phlogopite, chlorite, apatite and sulfide in individual inclusions. Fe-Ni alloys are present in serpentinized matrix in a spinel-rich zone.

\section{Geochemical Analyses and the Results}

\subsection{Analytical Methods}

We determined major- and trace-element compositions of minerals by using an electron probe micro analyzer (EPMA; EPMA-1720H, SHIMADZU) of Engineering Research Equipment Center at Kumamoto University, and a laser (193 nm ArF excimer; GeoLas Q-plus, MicroLas) ablation-inductively coupled plasma-mass spectrometer (Agilent 7500s, Agilent Technologies, Santa Clara, CA, USA) (LA-ICP-MS) at Kanazawa University, respectively. For the EPMA analyses, the accelerating voltage, prove current, and probe diameter were $15 \mathrm{kV}, 20 \mathrm{nA}$, and $5 \mu \mathrm{m}$, respectively. We used mineral and oxide standards $\left(\mathrm{SiO}_{2}, \mathrm{KTiPO}_{4}, \mathrm{NaAlSi}_{2} \mathrm{O}_{6}, \mathrm{Cr}_{2} \mathrm{O}_{3}, \mathrm{Fe}_{2} \mathrm{SiO}_{4}, \mathrm{MnO}, \mathrm{MgO}, \mathrm{CaSiO}_{3}\right.$, and $\mathrm{NiO}$ ) for calibration, and the counting time for each peak and background position was $10 \mathrm{~s}$ for both. For LA-ICP-MS analyses, we used $50 \mu \mathrm{m}$ diameter spot for clinopyroxene and $100 \mu \mathrm{m}$ diameter spot for orthopyroxene at a $6 \mathrm{~Hz}$ repetition rate with an energy density of $8 \mathrm{~J} / \mathrm{cm}^{2}$ per pulse. The NIST SRM 612 was used for external calibration standard and ${ }^{29} \mathrm{Si}$ as an internal standard, based on the $\mathrm{SiO}_{2}$ contents obtained from EPMA analyses. The details of analytical methods, including data precision and accuracy, were in Morishita et al. [36].

\subsection{Major-Element Compositions}

The results are shown in Figures 7-10 and Tables 2-5. We mainly used three parameters in following sections, $\mathrm{Mg} \#$ for all minerals, and $\mathrm{Cr} \#$ and $\mathrm{Y}_{\mathrm{Fe}}$ for chromian spinel, and are $\mathrm{Mg} /(\mathrm{Mg}+$ total $\mathrm{Fe})$ atomic ratio, $\mathrm{Cr} /(\mathrm{Cr}+\mathrm{Al})$ atomic ratio, and $\mathrm{Fe}^{3+} /\left(\mathrm{Al}+\mathrm{Cr}+\mathrm{Fe}^{3+}\right)$ atomic ratio, respectively. For calculation of $\mathrm{Mg \#}$ for chromian spinel, we calculated ferric iron content based on spinel stoichiometry and $\mathrm{Mg} \#$ for chromian spinel is $\mathrm{Mg} /\left(\mathrm{Mg}+\mathrm{Fe}^{2+}\right)$ atomic ratio. Orthopyroxene and clinopyroxene frequently occur as coarse porphyroclasts $(>1 \mathrm{~cm})$, and show no obvious chemical zoning. We also determined compositions of some amphiboles and used definition by Leake et al. [37] for their classification.

\subsubsection{LU Rocks (Dunite-Wehrlite-Clinopyroxenite of R1)}

Mg\# of olivine is almost constant (0.88-0.89) irrespective of the lithology (dunite, wehrlite, or clinopyroxenite) except for one dunite sample 2013MR2-0 ( $=0.91)$. NiO content of olivine varies from 0.14 to $0.27 \mathrm{wt} . \%$ and shows positive correlations with the Mg\# (Figure 7a; Table 2). Chromian spinel compositions also show almost constant Mg\# (0.36-0.46) and $\mathrm{Cr} \#(0.67-0.72)$ irrespective of the lithology (Figure 8a) and are similar to those in boninite or forearc peridotite. The $\mathrm{TiO}_{2}$ and $\mathrm{MnO}$ contents of chromian spinel are low, $0.12-0.19 \mathrm{wt} . \%$ and $0.30-0.49 \mathrm{wt} . \%$, respectively (Figure 8b; Table 3). The $\mathrm{Y}_{\mathrm{Fe}}$, of chromian spine, is also low and almost constant (0.07-0.12) (Figure 8c). Orthopyroxene, only observed in clinopyroxenite samples, shows the $\mathrm{Mg} \#$ almost the same as that of coexisting olivine $(\approx 0.89)$, and its $\mathrm{Al}_{2} \mathrm{O}_{3}, \mathrm{Cr}_{2} \mathrm{O}_{3}$ and $\mathrm{CaO}$ contents are almost constant, i.e., $0.89-1.03 \mathrm{wt} . \%$, $0.38-0.42 \mathrm{wt} . \%$, and $0.60-0.77 \mathrm{wt} . \%$, respectively (Figure 9; Table 4). The Mg\# of clinopyroxene is also constant, irrespective of lithology (0.92-0.93), except for one dunite (2013MR2-0) containing high-Mg\# olivine ( $\mathrm{Mg \#}=0.96$ ) (Figure 10; Table 5). $\mathrm{Al}_{2} \mathrm{O}_{3}$ and $\mathrm{Cr}_{2} \mathrm{O}_{3}$ contents are almost constant irrespective of lithology, 0.46-1.55 wt.\% and 0.15-0.95, respectively (Figure 10; Table 5). Tremolites occur as inclusion in chromian spinel in dunite or as discrete grains in clinopyroxenite (2013MR2-8 and 2013MR2-10). 
Table 2. Averaged values of major element compositions of olivine in each sample from the Mersin ophiolite.

\begin{tabular}{|c|c|c|c|c|c|c|c|c|c|c|c|c|c|c|c|c|}
\hline \multirow{3}{*}{$\begin{array}{c}\text { Rock Suite } \\
\text { Rock Type } \\
\text { Sample No. }\end{array}$} & \multicolumn{6}{|c|}{ Layered Ultramafic (LU) Rocks } & \multicolumn{9}{|c|}{ Moho Transition Zone (MTZ) Rocks } & \multirow{2}{*}{$\begin{array}{l}\begin{array}{l}\text { Mantle } \\
\text { Rocks }\end{array} \\
\text { Harz }\end{array}$} \\
\hline & Dunite & & Wehrlite & & Cpxite & & Dunite & & & & & & & & Harz & \\
\hline & 2013MR2-0 & 2013MR2-07 & 2013MR2-02 & 2013MR2-09 & 2013MR2-08 & 2013MR2-10 & 2014MR1-01 & 2014MR1-03 & 14T-MR03 & 2014MR1-04B & 2014MR1-05 & 2014MR1-06 & 2014MR1-09 & 2014MR1-14 & 2013MR1-02 & 2014MR2-16 \\
\hline $\begin{array}{c}\text { No. of } \\
\text { Analysis }\end{array}$ & $(\mathrm{N}=3)$ & $(\mathrm{N}=3)$ & $(\mathrm{N}=2)$ & $(\mathrm{N}=4)$ & $(\mathrm{N}=4)$ & $(N=5)$ & $(N=6)$ & $(N=6)$ & $(\mathrm{N}=3)$ & $(N=5)$ & $(\mathrm{N}=4)$ & $(N=6)$ & $(\mathrm{N}=5)$ & $(\mathrm{N}=3)$ & $(\mathrm{N}=1)$ & $(\mathrm{N}=2)$ \\
\hline $\mathrm{SiO}_{2}$ & 41.57 & 41.24 & 41.82 & 41.01 & 39.60 & 40.38 & 40.81 & 41.29 & 40.66 & 41.17 & 41.36 & 40.94 & 40.96 & 41.28 & 41.00 & 41.92 \\
\hline $\mathrm{TiO}_{2}$ & 0.00 & 0.00 & 0.00 & 0.00 & & & 0.00 & 0.01 & 0.03 & 0.00 & 0.00 & 0.00 & & 0.00 & 0.00 & 0.00 \\
\hline $\mathrm{Al}_{2} \mathrm{O}_{3}$ & 0.00 & 0.00 & 0.00 & 0.00 & & & 0.01 & & 0.03 & 0.01 & 0.00 & 0.01 & & 0.00 & 0.00 & 0.00 \\
\hline $\mathrm{Cr}_{2} \mathrm{O}_{3}$ & 0.01 & 0.01 & 0.02 & 0.01 & & & 0.01 & 0.02 & 0.03 & 0.01 & 0.01 & 0.01 & 0.01 & 0.00 & 0.00 & 0.01 \\
\hline $\mathrm{FeO}^{*}$ & 8.42 & 10.82 & 10.76 & 11.97 & 11.77 & 11.87 & 9.71 & 7.56 & 8.03 & 8.22 & 8.02 & 8.34 & 8.05 & 8.41 & 9.22 & 9.84 \\
\hline $\mathrm{MnO}$ & 0.12 & 0.12 & 0.14 & 0.16 & & & 0.13 & 0.11 & 0.16 & 0.12 & 0.11 & 0.12 & 0.11 & 0.11 & 0.09 & 0.13 \\
\hline $\mathrm{MgO}$ & 50.63 & 48.63 & 48.35 & 47.70 & 46.76 & 47.72 & 48.77 & 50.46 & 51.84 & 50.58 & 50.31 & 50.06 & 49.93 & 50.78 & 49.88 & 49.41 \\
\hline $\mathrm{CaO}$ & 0.05 & 0.02 & 0.02 & 0.02 & & & 0.06 & 0.06 & $\begin{array}{l}0.09 \\
0.09\end{array}$ & 0.06 & 0.08 & 0.08 & 0.06 & 0.06 & 0.00 & 0.02 \\
\hline $\mathrm{Na}_{2} \mathrm{O}$ & 0.00 & 0.00 & 0.00 & 0.00 & & & 0.01 & 0.01 & 0.01 & & 0.00 & & 0.01 & 0.00 & 0.00 & 0.00 \\
\hline $\mathrm{K}_{2} \mathrm{O}$ & 0.00 & 0.00 & 0.00 & 0.00 & & & 0.00 & 0.00 & 0.04 & 0.01 & 0.00 & 0.00 & & 0.00 & 0.00 & 0.00 \\
\hline $\mathrm{NiO}$ & 0.27 & 0.24 & 0.21 & 0.14 & & & 0.29 & 0.38 & 0.32 & 0.28 & 0.19 & 0.30 & 0.29 & 0.31 & 0.42 & 0.44 \\
\hline Total & 101.08 & 101.08 & 101.33 & 101.01 & 98.13 & 99.96 & 99.79 & 99.89 & 101.23 & 100.45 & 100.09 & 99.86 & 99.41 & 100.96 & 100.61 & 101.76 \\
\hline Mg\# & 0.915 & 0.889 & 0.889 & 0.877 & 0.876 & 0.878 & 0.900 & 0.922 & 0.920 & 0.916 & 0.918 & 0.914 & 0.917 & 0.915 & 0.906 & 0.899 \\
\hline Rock Suite & Mantle Rock & & & & & & & & & & & & & & & \\
\hline Rock Type & Harz & & & & (Harz Part) & Harz & & & & Dunite & & $\begin{array}{l}\text { (Dunite } \\
\text { Part) }\end{array}$ & $\begin{array}{l}\text { G } \\
\text { Chromitite }\end{array}$ & Wehrlite & Websterite & Opxite \\
\hline Sample No. & 2014MR2-15 & 2014MR2-13 & 2014MR2-12 & 2013MR7-01 & 2014MR2-09 & 2014MR2-06 & 2014MR2-03 & 2014MR2-01 & 2013MR8-01 & 2014MR2-14 & 2014MR2-11 & 2014MR2-09 & 14T-MR12(p) & 14T-MR04 & 2013MR5-02 & 2013MR4-02 \\
\hline $\begin{array}{c}\text { No. of } \\
\text { Analysis }\end{array}$ & $(\mathrm{N}=4)$ & $(\mathrm{N}=5)$ & $(\mathrm{N}=4)$ & $(\mathrm{N}=3)$ & $(\mathrm{N}=3)$ & $(\mathrm{N}=5)$ & $(\mathrm{N}=2)$ & $(\mathrm{N}=2)$ & $(\mathrm{N}=4)$ & $(\mathrm{N}=3)$ & $(\mathrm{N}=3)$ & $(\mathrm{N}=1)$ & $(\mathrm{N}=2)$ & $(\mathrm{N}=4)$ & $(\mathrm{N}=3)$ & $(\mathrm{N}=4)$ \\
\hline $\mathrm{SiO}_{2}$ & 42.10 & 41.24 & 41.80 & 41.09 & 40.61 & 41.08 & 40.89 & 41.01 & 41.86 & 41.19 & 41.62 & 41.33 & 40.62 & 40.41 & 41.23 & 41.42 \\
\hline $\mathrm{TiO}_{2}$ & 0.00 & 0.00 & 0.00 & 0.01 & 0.00 & 0.01 & 0.01 & 0.00 & 0.00 & 0.00 & 0.00 & 0.00 & 0.01 & 0.02 & 0.00 & 0.00 \\
\hline $\mathrm{Al}_{2} \mathrm{O}_{3}$ & 0.00 & 0.00 & 0.00 & 0.01 & 0.00 & 0.00 & 0.01 & 0.00 & 0.00 & 0.00 & 0.00 & 0.01 & 0.01 & 0.03 & 0.00 & 0.00 \\
\hline $\mathrm{Cr}_{2} \mathrm{O}_{3}$ & 0.01 & 0.01 & 0.01 & 0.00 & 0.01 & 0.02 & 0.01 & 0.02 & 0.00 & 0.02 & 0.01 & 0.00 & 0.43 & 0.04 & 0.00 & 0.00 \\
\hline $\mathrm{FeO}^{*}$ & 9.80 & 9.78 & 8.81 & 9.81 & 9.55 & 8.56 & 9.86 & 8.97 & 9.40 & 7.78 & 8.21 & 9.87 & 3.99 & 9.41 & 9.10 & 9.62 \\
\hline $\mathrm{MnO}$ & 0.14 & 0.11 & 0.10 & 0.09 & 0.10 & 0.10 & 0.11 & 0.10 & 0.11 & 0.10 & 0.08 & 0.13 & 0.09 & & 0.12 & 0.13 \\
\hline $\mathrm{MgO}$ & 49.97 & 50.27 & 50.25 & 48.71 & 49.22 & 50.43 & 49.97 & 50.22 & 50. & 51.49 & 50. & 49.05 & 54.01 & 50.58 & 49.30 & 49.15 \\
\hline $\mathrm{CaO}$ & 0.01 & 0. & 0.01 & 0.02 & 0.01 & 0.1 & 0.0 & 0.01 & 0.01 & 0.09 & 0.0 & 0.01 & 0.07 & 0.04 & 0.01 & 0.04 \\
\hline $\mathrm{Na}_{2} \mathrm{O}$ & 0.00 & 0.00 & 0.00 & 0.00 & 0.01 & 0.00 & 0.00 & 0.00 & 0.00 & 0.00 & 0.00 & 0.01 & 0.02 & 0.02 & 0.00 & 0.00 \\
\hline $\mathrm{K}_{2} \mathrm{O}$ & 0.00 & 0.00 & 0.00 & 0.00 & 0.00 & 0.00 & 0.00 & 0.00 & 0.00 & 0.00 & 0.00 & 0.01 & 0.02 & 0.02 & 0.00 & 0.00 \\
\hline $\mathrm{NiO}$ & 0.39 & 0.45 & 0.44 & 0.41 & 0.39 & $\begin{array}{l}0.40 \\
0.42\end{array}$ & 0.37 & 0.37 & 0.42 & 0.43 & 0.40 & 0.42 & 0.58 & $\begin{array}{l}0.02 \\
0.20\end{array}$ & 0.41 & 0.39 \\
\hline Total & 102.42 & 101.88 & 101.42 & 100.15 & 99.89 & 100.64 & 101.22 & 100.70 & 101.82 & 101.10 & 100.93 & 100.84 & 99.83 & 100.78 & 100.17 & 100.76 \\
\hline Mg\# & 0.901 & 0.902 & 0.910 & 0.898 & 0.902 & 0.913 & 0.900 & 0.909 & 0.905 & 0.922 & 0.917 & 0.899 & 0.960 & 0.905 & 0.906 & 0.901 \\
\hline
\end{tabular}

If there are different lithological parts within one sample, it is noted, e.g., (Harz part). Abbreviations of rock lith
assuming all iron is $\mathrm{Fe}^{2+}$. $\mathrm{Mg \#}, \mathrm{Mg} /(\mathrm{Mg}+\mathrm{Fe}$ total) atomic ratio. Blank means below their detection limits. 
Table 3. Averaged values of major element compositions of chromian spinel in each sample from the Mersin ophiolite.

\begin{tabular}{|c|c|c|c|c|c|c|c|c|c|c|c|c|c|c|c|c|}
\hline Rock Suite & LU Rocks & & & & MTZ Rocks & & & & & & & & & & Mantle Rocks & \\
\hline Rock Type & Dunite & & Wehrlite & & Dunite & & & & & & & & M Chromitite & Harz & Harz & \\
\hline Sample No. & 2013MR2-0 & 2013MR2-07 & 2013MR2-02 & 2013MR2-09 & 2014MR1-01 & 2014MR1-03 & 14T-MR03 & 2014MR1-04 & B2014MR1-05 & 2014MR1-06 & 2014MR1-09 & 2014MR1-14 & 14T-MR01 & 2013MR1-02 & 2014MR2-16 & 2014MR2-13 \\
\hline $\begin{array}{c}\text { No. of } \\
\text { Analysis }\end{array}$ & $(N=7)$ & $(\mathrm{N}=6)$ & $(N=6)$ & $(\mathrm{N}=2)$ & $(\mathrm{N}=8)$ & $(N=7)$ & $(\mathrm{N}=3)$ & $(N=6)$ & $(\mathrm{N}=3)$ & $(\mathrm{N}=6)$ & $(N=5)$ & $(\mathrm{N}=6)$ & $(N=7)$ & $(\mathrm{N}=6)$ & $(\mathrm{N}=2)$ & $(\mathrm{N}=3)$ \\
\hline $\mathrm{SiO}_{2}$ & 0.04 & 0.04 & 0.03 & 0.05 & 0.04 & 0.02 & 0.08 & 0.05 & 0.03 & 0.16 & 0.01 & 0.02 & 0.07 & 0.05 & 0.06 & 0.02 \\
\hline $\mathrm{TiO}_{2}$ & 0.18 & 0.18 & 0.18 & 0.13 & 0.14 & 0.14 & 0.17 & 0.17 & 0.17 & 0.08 & 0.11 & 0.16 & 0.20 & 0.05 & 0.02 & 0.02 \\
\hline $\mathrm{Al}_{2} \mathrm{O}_{3}$ & 13.45 & 14.65 & 14.33 & 14.37 & 10.35 & 8.78 & 9.35 & 9.58 & 9.60 & 4.84 & 7.03 & 9.20 & 10.21 & 13.67 & 10.94 & 12.11 \\
\hline $\mathrm{Cr}_{2} \mathrm{O}_{3}$ & 49.04 & 49.66 & 49.43 & 48.39 & 53.38 & 56.73 & 57.10 & 56.08 & 55.68 & 60.08 & 57.22 & 56.82 & 60.22 & 53.53 & 55.06 & 56.14 \\
\hline $\mathrm{Fe}_{2} \mathrm{O}_{3}$ & 7.16 & 5.36 & 5.61 & 6.67 & 6.08 & 4.75 & 4.42 & 5.21 & 4.92 & 5.99 & 5.92 & 4.84 & 2.21 & 3.17 & 4.09 & 3.20 \\
\hline $\mathrm{FeO}^{*}$ & 21.17 & 21.38 & 22.12 & 22.80 & 21.12 & 19.84 & 19.97 & 20.18 & 20.35 & 21.31 & 21.14 & 19.92 & 13.66 & 19.18 & 20.12 & 19.61 \\
\hline $\mathrm{MnO}$ & 0.37 & 0.38 & 0.39 & 0.41 & 0.41 & 0.38 & 0.30 & 0.41 & 0.37 & 0.47 & 0.41 & 0.39 & 0.16 & 0.31 & 0.36 & 0.31 \\
\hline $\mathrm{MgO}$ & 8.37 & 8.45 & 7.91 & 7.50 & 8.03 & 8.69 & 8.88 & 8.78 & 8.51 & 7.45 & 7.67 & 8.87 & 13.06 & 9.68 & 8.68 & 9.50 \\
\hline $\mathrm{CaO}$ & 0.00 & 0.07 & 0.02 & 0.01 & 0.01 & 0.02 & 0.02 & 0.01 & 0.00 & 0.01 & 0.00 & 0.00 & 0.03 & 0.01 & 0.02 & 0.00 \\
\hline $\mathrm{Na}_{2} \mathrm{O}$ & 0.00 & 0.00 & 0.00 & 0.00 & 0.00 & 0.00 & 0.06 & 0.00 & 0.00 & 0.00 & 0.00 & 0.00 & 0.06 & 0.00 & 0.00 & 0.00 \\
\hline $\mathrm{K}_{2} \mathrm{O}$ & 0.00 & 0.00 & 0.00 & 0.00 & 0.00 & 0.01 & 0.01 & 0.00 & 0.00 & 0.00 & 0.01 & 0.00 & 0.01 & 0.00 & 0.00 & 0.00 \\
\hline $\mathrm{NiO}$ & 0.07 & 0.06 & 0.04 & 0.03 & 0.05 & 0.09 & 0.08 & 0.03 & 0.02 & 0.03 & 0.03 & 0.07 & 0.11 & 0.06 & 0.08 & 0.03 \\
\hline Total & 99.84 & 100.22 & 100.04 & 100.34 & 99.61 & 99.46 & 100.45 & 100.49 & 99.66 & 100.42 & 99.55 & 100.29 & 100.01 & 99.71 & 99.42 & 100.95 \\
\hline Mg\# & 0.414 & 0.413 & 0.389 & 0.370 & 0.404 & 0.439 & 0.442 & 0.437 & 0.427 & 0.384 & 0.393 & 0.442 & 0.630 & 0.473 & 0.435 & 0.463 \\
\hline Cr\# & 0.710 & 0.695 & 0.698 & 0.693 & 0.776 & 0.812 & 0.804 & 0.797 & 0.795 & 0.893 & 0.845 & 0.806 & 0.798 & 0.724 & 0.772 & 0.757 \\
\hline $\mathrm{Y}_{\mathrm{Cr}}$ & 0.646 & 0.648 & 0.649 & 0.635 & 0.716 & 0.763 & 0.759 & 0.745 & 0.746 & 0.823 & 0.780 & 0.756 & 0.776 & 0.696 & 0.732 & 0.727 \\
\hline $\mathrm{Y}_{\mathrm{Al}}$ & 0.264 & 0.285 & 0.281 & 0.281 & 0.207 & 0.176 & 0.185 & 0.190 & 0.192 & 0.099 & 0.143 & 0.183 & 0.196 & 0.265 & 0.217 & 0.234 \\
\hline $\mathrm{Y}_{\mathrm{Fe}}$ & 0.090 & 0.067 & 0.070 & 0.083 & 0.078 & 0.061 & 0.056 & 0.066 & 0.063 & 0.078 & 0.077 & 0.061 & 0.027 & 0.039 & 0.052 & 0.039 \\
\hline Rock Suite & Mantle rocks & & & & & & & & & & & & & & & \\
\hline Rock Type & Harz & & (Harz part) & Harz & & & & Dunite & & $\begin{array}{l}\text { (Dunite } \\
\text { part) }\end{array}$ & G Chromitite & & Wehrlite & Websterite & Opxite & \\
\hline Sample No. & 2014MR2-12 & 2013MR7-01 & 2014MR2-09 & 2014MR2-06 & 2014MR2-03 & 2014MR2-01 & 2013MR8-01 & 2014MR2-14 & 2014MR2-11 & 2014MR2-09 & $\begin{array}{c}\text { 14T-MR12 } \\
\text { (p) }\end{array}$ & $\begin{array}{c}\text { 14T-MR12 } \\
\text { (r) }\end{array}$ & 14T-MR04 & 2013MR5-02 & 2013MR4-02 & \\
\hline $\begin{array}{c}\text { No. of } \\
\text { Analysis }\end{array}$ & $(\mathrm{N}=2)$ & $(\mathrm{N}=2)$ & $(\mathrm{N}=2)$ & $(\mathrm{N}=5)$ & $(\mathrm{N}=4)$ & $(\mathrm{N}=2)$ & $(\mathrm{N}=4)$ & $(\mathrm{N}=5)$ & $(\mathrm{N}=1)$ & $(\mathrm{N}=2)$ & $(\mathrm{N}=4)$ & $(\mathrm{N}=4)$ & $(N=7)$ & $(\mathrm{N}=2)$ & $(\mathrm{N}=3)$ & \\
\hline $\mathrm{SiO}_{2}$ & 0.01 & 0.04 & 0.04 & 0.02 & 0.03 & 0.04 & 0.02 & 0.02 & 0.52 & 0.03 & 0.08 & 0.06 & 0.10 & 0.02 & 0.09 & \\
\hline $\mathrm{TiO}_{2}$ & 0.05 & 0.02 & 0.04 & 0.04 & 0.03 & 0.07 & 0.05 & 0.19 & 0.12 & 0.05 & 0.20 & 0.21 & 0.20 & 0.11 & 0.08 & \\
\hline $\mathrm{Al}_{2} \mathrm{O}_{3}$ & 20.19 & 17.25 & 13.47 & 12.79 & 12.86 & 16.67 & 16.15 & 13.05 & 10.21 & 12.99 & 10.83 & 10.92 & 9.76 & 15.72 & 11.71 & \\
\hline $\mathrm{Cr}_{2} \mathrm{O}_{3}$ & 48.39 & 48.05 & 53.83 & 55.01 & 54.45 & 50.71 & 52.27 & 54.24 & 56.40 & 52.28 & 58.16 & 58.67 & 57.78 & 51.69 & 55.33 & \\
\hline $\mathrm{Fe}_{2} \mathrm{O}_{3}$ & 2.02 & 4.84 & 3.23 & 2.87 & 3.36 & 2.98 & 2.38 & 3.57 & 2.98 & 4.28 & 2.96 & 2.89 & 2.54 & 2.68 & 3.22 & \\
\hline $\mathrm{FeO}^{*}$ & 18.36 & 19.53 & 19.28 & 18.92 & 19.95 & 17.99 & 18.29 & 17.70 & 18.25 & 21.33 & 14.14 & 13.25 & 20.01 & 18.17 & 21.12 & \\
\hline $\mathrm{MnO}$ & 0.28 & 0.31 & 0.28 & 0.34 & 0.32 & 0.31 & 0.32 & 0.32 & 0.39 & 0.37 & 0.17 & 0.16 & 0.28 & 0.32 & 0.40 & \\
\hline $\mathrm{MgO}$ & 11.07 & 9.84 & 9.63 & 9.79 & 9.21 & 10.76 & 10.63 & 10.66 & 9.61 & 8.15 & 12.67 & 13.33 & 8.77 & 10.49 & 8.33 & \\
\hline $\mathrm{CaO}$ & 0.00 & 0.00 & 0.01 & 0.03 & 0.00 & 0.02 & 0.01 & 0.00 & 0.01 & 0.00 & 0.03 & 0.02 & 0.04 & 0.00 & 0.01 & \\
\hline $\mathrm{Na}_{2} \mathrm{O}$ & 0.00 & 0.00 & 0.00 & 0.00 & 0.00 & 0.00 & 0.00 & 0.00 & 0.00 & 0.00 & 0.05 & 0.04 & 0.05 & 0.00 & 0.00 & \\
\hline $\mathrm{K}_{2} \mathrm{O}$ & 0.00 & 0.00 & 0.00 & 0.00 & 0.00 & 0.00 & 0.00 & 0.00 & 0.03 & 0.00 & 0.02 & 0.03 & 0.02 & 0.00 & 0.00 & \\
\hline $\mathrm{NiO}$ & 0.08 & 0.09 & 0.09 & 0.06 & 0.09 & 0.05 & 0.04 & 0.10 & 0.06 & 0.05 & 0.14 & 0.08 & 0.08 & 0.03 & 0.05 & \\
\hline Total & 100.43 & 99.97 & 99.90 & 99.86 & 100.29 & 99.59 & 100.15 & 99.85 & 98.56 & 99.52 & 99.44 & 99.65 & 99.64 & 99.24 & 100.34 & \\
\hline Mg\# & 0.518 & 0.473 & 0.471 & 0.480 & 0.451 & 0.516 & 0.509 & 0.518 & $\begin{array}{l}0.000 \\
0.484\end{array}$ & 0.405 & $\begin{array}{l}0.615 \\
0.615\end{array}$ & 0.642 & $\begin{array}{l}0.04 \\
0.439\end{array}$ & $\begin{array}{l}0.507 \\
0.507\end{array}$ & 0.413 & \\
\hline Cr\# & 0.617 & 0.651 & 0.728 & 0.743 & 0.740 & 0.671 & 0.685 & 0.736 & 0.788 & 0.730 & 0.783 & 0.783 & 0.799 & 0.688 & 0.760 & \\
\hline $\mathrm{Y}_{\mathrm{Cr}}$ & 0.602 & 0.613 & 0.699 & 0.716 & 0.709 & 0.647 & 0.665 & 0.704 & 0.758 & 0.690 & 0.754 & 0.755 & 0.773 & 0.665 & 0.730 & \\
\hline $\mathrm{Y}_{\mathrm{Al}}$ & 0.374 & 0.328 & 0.261 & 0.248 & 0.250 & 0.317 & 0.306 & 0.252 & 0.204 & 0.256 & 0.209 & 0.210 & 0.195 & 0.302 & 0.230 & \\
\hline $\mathrm{Y}_{\mathrm{Fe}}$ & 0.024 & 0.059 & 0.040 & 0.036 & 0.042 & 0.036 & 0.029 & 0.044 & 0.038 & 0.054 & 0.036 & 0.035 & 0.032 & 0.033 & 0.040 & \\
\hline
\end{tabular}

Banded chromitite,14T-MR12, showing graded bedding and is separated two parts, spinel-poor part (14T-MR12(p)) and spinel-rich part (14T-MR12(r)), respectively. If there are different lithological parts within one sample, it is noted, e.g., (Harz part). Abbreviations of rock lithology are the same as Figure 2. FeO* is total iron oxide content calculated by assuming all iron is $\mathrm{Fe}^{2+} . \mathrm{Mg}, \mathrm{Mg} /(\mathrm{Mg}+\mathrm{Fe}$ total $)$ atomic ratio. $\mathrm{Mg} \#, \mathrm{Mg} /\left(\mathrm{Mg}+\mathrm{Fe}^{2+}\right)$ atomic ratio, $\mathrm{Cr}, \mathrm{Cr} /(\mathrm{Cr}+\mathrm{Al})$ atomic ratio; $\mathrm{Y}_{\mathrm{Al}}, \mathrm{Al} /\left(\mathrm{Al}+\mathrm{Cr}+\mathrm{Fe}{ }^{3+}\right)$ atomic ratio; $\mathrm{Y}_{\mathrm{Cr}}, \mathrm{Al} /\left(\mathrm{Al}+\mathrm{Cr}+\mathrm{Fe}{ }^{3+}\right)$ atomic ratio; $\mathrm{Y}_{\mathrm{Fe}}, \mathrm{Al} /\left(\mathrm{Al}+\mathrm{Cr}+\mathrm{Fe}^{3+}\right)$ atomic ratio; $\mathrm{M}$ chromitite, massive chromitite; $\mathrm{G}$ chromitite, graded chromitite. 
Table 4. Averaged values of major element compositions of orthopyroxene in each sample from the Mersin ophiolite.

\begin{tabular}{|c|c|c|c|c|c|c|c|c|c|c|c|c|c|c|}
\hline Rock Suite & LU Rocks & & MTZ Rocks & Mantle Rocks & & & & & & & & & & \\
\hline Rock Type & Cpxite & & Harz & Harz & & & & & (Harz Part) & Harz & & & & Websterite \\
\hline Sample No. & 2013MR2-08 & 2013MR2-10 & 2013MR1-02 & 2014MR2-16 & 2014MR2-15 & 2014MR2-13 & 2014MR2-12 & 2013MR7-01 & 2014MR2-09 & 2014MR2-06 & 2014MR2-03 & 2014MR2-01 & 2013MR8-01 & 2013MR5-02 \\
\hline $\begin{array}{c}\text { No. of } \\
\text { Analysis }\end{array}$ & $(\mathrm{N}=4)$ & $(\mathrm{N}=2)$ & $(\mathrm{N}=12)$ & $(\mathrm{N}=2)$ & $(\mathrm{N}=2)$ & $(\mathrm{N}=6)$ & $(\mathrm{N}=4)$ & $(\mathrm{N}=4)$ & $(\mathrm{N}=4)$ & $(\mathrm{N}=4)$ & $(\mathrm{N}=5)$ & $(\mathrm{N}=3)$ & $(\mathrm{N}=3)$ & $(\mathrm{N}=3)$ \\
\hline $\mathrm{SiO}_{2}$ & 56.13 & 55.81 & 58.15 & 57.49 & 58.54 & 57.25 & 57.34 & 57.96 & 57.08 & 57.52 & 57.70 & 57.68 & 57.66 & 57.24 \\
\hline $\mathrm{TiO}_{2}$ & & & 0.01 & 0.00 & 0.01 & 0.00 & 0.01 & 0.00 & 0.01 & 0.01 & 0.00 & 0.02 & 0.01 & 0.02 \\
\hline $\mathrm{Al}_{2} \mathrm{O}_{3}$ & 0.89 & 0.97 & 1.00 & 0.76 & 0.86 & 0.83 & 1.55 & 1.14 & 0.99 & 0.77 & 0.83 & 1.09 & 1.34 & 1.07 \\
\hline $\mathrm{Cr}_{2} \mathrm{O}_{3}$ & 0.44 & 0.48 & 0.46 & 0.38 & 0.31 & 0.37 & 0.51 & 0.48 & 0.43 & 0.34 & 0.38 & 0.36 & 0.54 & 0.44 \\
\hline $\mathrm{FeO}^{*}$ & 7.65 & 7.53 & 6.09 & 6.23 & 6.59 & 6.31 & 5.98 & 6.49 & 6.27 & 5.78 & 6.40 & 5.90 & 6.07 & 5.85 \\
\hline $\mathrm{MnO}$ & & & 0.13 & 0.14 & 0.15 & 0.13 & 0.10 & 0.11 & 0.14 & 0.11 & 0.13 & 0.13 & 0.13 & 0.14 \\
\hline $\mathrm{MgO}$ & 33.36 & 33.05 & 34.53 & 34.02 & 34.81 & 34.87 & 34.07 & 33.87 & 33.86 & 34.98 & 34.78 & 34.49 & 34.11 & 33.97 \\
\hline $\mathrm{CaO}$ & 0.74 & 0.70 & 0.94 & 1.11 & 0.60 & 0.83 & 1.01 & 0.83 & 0.93 & 0.95 & 1.22 & 0.74 & 1.21 & 0.84 \\
\hline $\mathrm{Na}_{2} \mathrm{O}$ & & & 0.00 & 0.01 & 0.00 & 0.00 & 0.01 & 0.01 & 0.01 & 0.01 & 0.01 & 0.00 & 0.01 & 0.00 \\
\hline $\mathrm{K}_{2} \mathrm{O}$ & & & 0.00 & 0.01 & 0.00 & 0.00 & 0.00 & 0.01 & 0.01 & 0.00 & 0.00 & 0.00 & 0.00 & 0.00 \\
\hline $\mathrm{NiO}$ & & & 0.11 & 0.11 & 0.07 & 0.10 & 0.07 & 0.12 & 0.12 & 0.10 & 0.11 & 0.09 & 0.11 & 0.10 \\
\hline Total & 99.21 & 98.54 & 101.41 & 100.26 & 101.94 & 100.70 & 100.66 & 101.01 & 99.85 & 100.57 & 101.56 & 100.49 & 101.19 & 99.67 \\
\hline Mg\# & 0.886 & 0.887 & 0.910 & 0.907 & 0.904 & 0.908 & 0.910 & 0.903 & 0.906 & 0.915 & 0.906 & 0.912 & 0.909 & 0.912 \\
\hline
\end{tabular}

If there are different lithological parts within one sample, it is noted, e.g., (Harz part). Abbreviations of rock lithology are the same as Figure 2. FeO* is total iron oxide content calculated by assuming all iron is $\mathrm{Fe}^{2+}$. $\mathrm{Mg \#}, \mathrm{Mg} /(\mathrm{Mg}+\mathrm{Fe}$ total $)$ atomic ratio. Blank means below their detection limits. 
Table 5. Averaged values of major element compositions of clinopyroxene in each sample from the Mersin ophiolite.

\begin{tabular}{|c|c|c|c|c|c|c|c|c|c|c|c|c|c|c|}
\hline Rock Suite & LU Rocks & & & & & & MTZ Rocks & & & & & & & $\begin{array}{l}\text { Mantle } \\
\text { Rocks }\end{array}$ \\
\hline Rock Type & Dunite & & Wehrlite & & Cpxite & & Dunite & & & & & & Harz & Harz \\
\hline Sample No. & 2013MR2-0 & 2013MR2-07 & 2013MR2-02 & 2013MR2-09 & 2013MR2-08 & 2013MR2-10 & 2014MR1-01 & 2014MR1-03 & 2014MR1-05 & 2014MR1-06 & 2014MR1-09 & 2014MR1-14 & 2013MR1-02 & 2014MR2-16 \\
\hline $\begin{array}{c}\text { No. of } \\
\text { Analysis }\end{array}$ & $(\mathrm{N}=6)$ & $(\mathrm{N}=5)$ & $(\mathrm{N}=5)$ & $(N=7)$ & $(N=5)$ & $(\mathrm{N}=5)$ & $(\mathrm{N}=3)$ & $(\mathrm{N}=2)$ & $(\mathrm{N}=1)$ & $(\mathrm{N}=3)$ & $(\mathrm{N}=1)$ & $(\mathrm{N}=1)$ & $(\mathrm{N}=9)$ & $(\mathrm{N}=2)$ \\
\hline $\mathrm{SiO}_{2}$ & 55.61 & 55.11 & 54.75 & 54.74 & 53.73 & 53.57 & 54.67 & 54.65 & 55.48 & 54.59 & 53.42 & 53.88 & 55.58 & 55.96 \\
\hline $\mathrm{TiO}_{2}$ & 0.04 & 0.03 & 0.03 & 0.04 & & & 0.03 & 0.04 & 0.01 & 0.01 & 0.04 & 0.05 & 0.01 & 0.00 \\
\hline $\mathrm{Al}_{2} \mathrm{O}_{3}$ & 0.83 & 0.82 & 1.11 & 1.03 & 1.02 & 0.98 & 0.47 & 0.69 & 0.28 & 0.36 & 0.42 & 0.79 & 0.94 & 0.74 \\
\hline $\mathrm{Cr}_{2} \mathrm{O}_{3}$ & 0.54 & 0.38 & 0.68 & 0.53 & 0.72 & 0.65 & 0.18 & 0.46 & 0.12 & 0.45 & 0.35 & 0.35 & 0.55 & 0.51 \\
\hline $\mathrm{FeO}^{*}$ & 1.41 & 2.24 & 2.84 & 2.52 & 2.53 & 2.69 & 1.57 & 1.31 & 1.48 & 1.50 & 1.23 & 1.32 & 2.01 & 1.92 \\
\hline $\mathrm{MnO}$ & 0.04 & 0.07 & 0.09 & 0.09 & & & 0.03 & 0.01 & 0.00 & 0.04 & 0.04 & 0.01 & 0.06 & 0.04 \\
\hline $\mathrm{MgO}$ & 17.37 & 17.53 & 17.50 & 17.49 & 17.72 & 18.28 & 17.47 & 17.25 & 18.30 & 17.52 & 17.41 & 17.31 & 17.79 & 17.68 \\
\hline $\mathrm{CaO}$ & 25.46 & 24.43 & 23.87 & 23.80 & 23.73 & 23.09 & 25.17 & 24.75 & 24.42 & 24.57 & 25.12 & 25.35 & 24.08 & 24.27 \\
\hline $\mathrm{Na}_{2} \mathrm{O}$ & 0.08 & 0.05 & 0.09 & 0.07 & & & 0.12 & 0.11 & 0.17 & 0.08 & 0.07 & 0.18 & 0.08 & 0.06 \\
\hline $\mathrm{K}_{2} \mathrm{O}$ & 0.00 & 0.00 & 0.00 & 0.00 & & & 0.00 & & 0.00 & 0.00 & 0.00 & 0.00 & 0.00 & 0.00 \\
\hline $\mathrm{NiO}$ & 0.04 & 0.03 & 0.03 & 0.04 & & & 0.04 & 0.04 & 0.08 & 0.05 & 0.04 & 0.09 & 0.07 & 0.03 \\
\hline Total & 101.42 & 100.69 & 100.99 & 100.36 & 99.43 & 99.26 & 99.75 & 99.31 & 100.35 & 99.17 & 98.14 & 99.34 & 101.17 & 101.22 \\
\hline Mg\# & 0.956 & 0.933 & 0.917 & 0.925 & 0.926 & 0.924 & 0.952 & 0.959 & 0.957 & 0.954 & 0.962 & 0.959 & 0.940 & 0.943 \\
\hline Rock Suite & Mantle Rocks & & & & & & & & & & & & & \\
\hline Rock Type & Harz & & & & (Harz Part) & Harz & & & & Dunite & $\begin{array}{l}\text { (Dunite } \\
\text { Part) }\end{array}$ & Wehrlite & Websterite & Opxite \\
\hline Sample No. & 2014MR2-15 & 2014MR2-13 & 2014MR2-12 & 2013MR7-01 & 2014MR2-09 & 2014MR2-06 & 2014MR2-03 & 2014MR2-01 & 2013MR8-01 & 2014MR2-11 & 2014MR2-09 & 14T-MR04 & 2013MR5-02 & 2013MR4-02 \\
\hline $\begin{array}{l}\text { No. of } \\
\text { Analysis }\end{array}$ & $(\mathrm{N}=1)$ & $(\mathrm{N}=3)$ & $(\mathrm{N}=8)$ & $(\mathrm{N}=3)$ & $(\mathrm{N}=6)$ & $(\mathrm{N}=3)$ & $(\mathrm{N}=3)$ & $(\mathrm{N}=2)$ & $(\mathrm{N}=10)$ & $(\mathrm{N}=2)$ & $(\mathrm{N}=1)$ & $(\mathrm{N}=4)$ & $(\mathrm{N}=6)$ & $(\mathrm{N}=4)$ \\
\hline $\mathrm{SiO}_{2}$ & 54.22 & 54.41 & 54.18 & 55.13 & 54.38 & 53.37 & 53.99 & 54.51 & 55.10 & 55.06 & 55.16 & 53.69 & 54.19 & 55.04 \\
\hline $\mathrm{TiO}_{2}$ & 0.00 & 0.01 & 0.01 & 0.01 & 0.00 & 0.01 & 0.01 & 0.02 & 0.02 & 0.02 & 0.00 & 0.07 & 0.02 & 0.01 \\
\hline $\mathrm{Al}_{2} \mathrm{O}_{3}$ & 0.83 & 0.83 & 1.37 & 0.95 & 0.80 & 0.72 & 0.78 & 0.93 & 1.10 & 0.79 & 0.10 & 0.65 & 0.91 & 0.75 \\
\hline $\mathrm{Cr}_{2} \mathrm{O}_{3}$ & 0.46 & 0.46 & 0.61 & 0.45 & 0.42 & 0.47 & 0.43 & 0.41 & 0.59 & 0.60 & 0.07 & 0.59 & 0.56 & 0.43 \\
\hline $\mathrm{FeO}^{*}$ & 2.82 & 2.02 & 1.87 & 1.93 & 1.89 & 1.93 & 1.95 & 1.76 & 1.80 & 1.70 & 1.26 & 1.89 & 1.96 & 1.96 \\
\hline $\mathrm{MnO}$ & 0.09 & 0.07 & 0.05 & 0.07 & 0.07 & 0.05 & 0.08 & 0.06 & 0.06 & 0.05 & 0.04 & 0.13 & 0.06 & 0.05 \\
\hline $\mathrm{MgO}$ & 20.34 & 18.05 & 18.00 & 17.89 & 17.93 & 17.91 & 18.21 & 17.78 & 17.87 & 17.55 & 17.75 & 18.22 & 18.06 & 17.75 \\
\hline $\mathrm{CaO}$ & 21.02 & 24.56 & 23.95 & 24.15 & 24.20 & 23.90 & 24.67 & 23.95 & 24.49 & 24.19 & 25.47 & 24.56 & 23.25 & 24.30 \\
\hline $\mathrm{Na}_{2} \mathrm{O}$ & 0.13 & 0.05 & 0.05 & 0.05 & 0.06 & 0.13 & 0.05 & 0.07 & 0.05 & 0.17 & 0.00 & 0.07 & 0.11 & 0.16 \\
\hline $\mathrm{K}_{2} \mathrm{O}$ & 0.00 & 0.00 & 0.00 & 0.01 & 0.00 & 0.00 & 0.00 & 0.01 & 0.00 & 0.00 & 0.00 & 0.04 & 0.00 & 0.00 \\
\hline $\mathrm{NiO}$ & 0.02 & 0.06 & 0.07 & 0.06 & 0.05 & 0.03 & 0.05 & 0.04 & 0.05 & 0.05 & 0.07 & 0.11 & 0.06 & 0.05 \\
\hline Total & 99.93 & 100.51 & 100.16 & 100.70 & 99.79 & 98.53 & 100.22 & 99.54 & 101.13 & 100.18 & 99.91 & 100.00 & 99.19 & 100.50 \\
\hline Mg\# & 0.928 & 0.941 & 0.945 & 0.943 & 0.942 & 0.943 & 0.943 & 0.947 & 0.947 & 0.948 & 0.962 & 0.945 & 0.943 & 0.942 \\
\hline
\end{tabular}

If there are different lithological parts within one sample, it is noted, e.g., (Harz part). Abbreviations of rock lithology are the same as Figure 2. FeO* is total iron oxide content calculated by assuming all iron is $\mathrm{Fe}^{2+}$. $\mathrm{Mg} \#, \mathrm{Mg} /(\mathrm{Mg}+\mathrm{Fe}$ total $)$ atomic ratio. Blank means below their detection limits. 

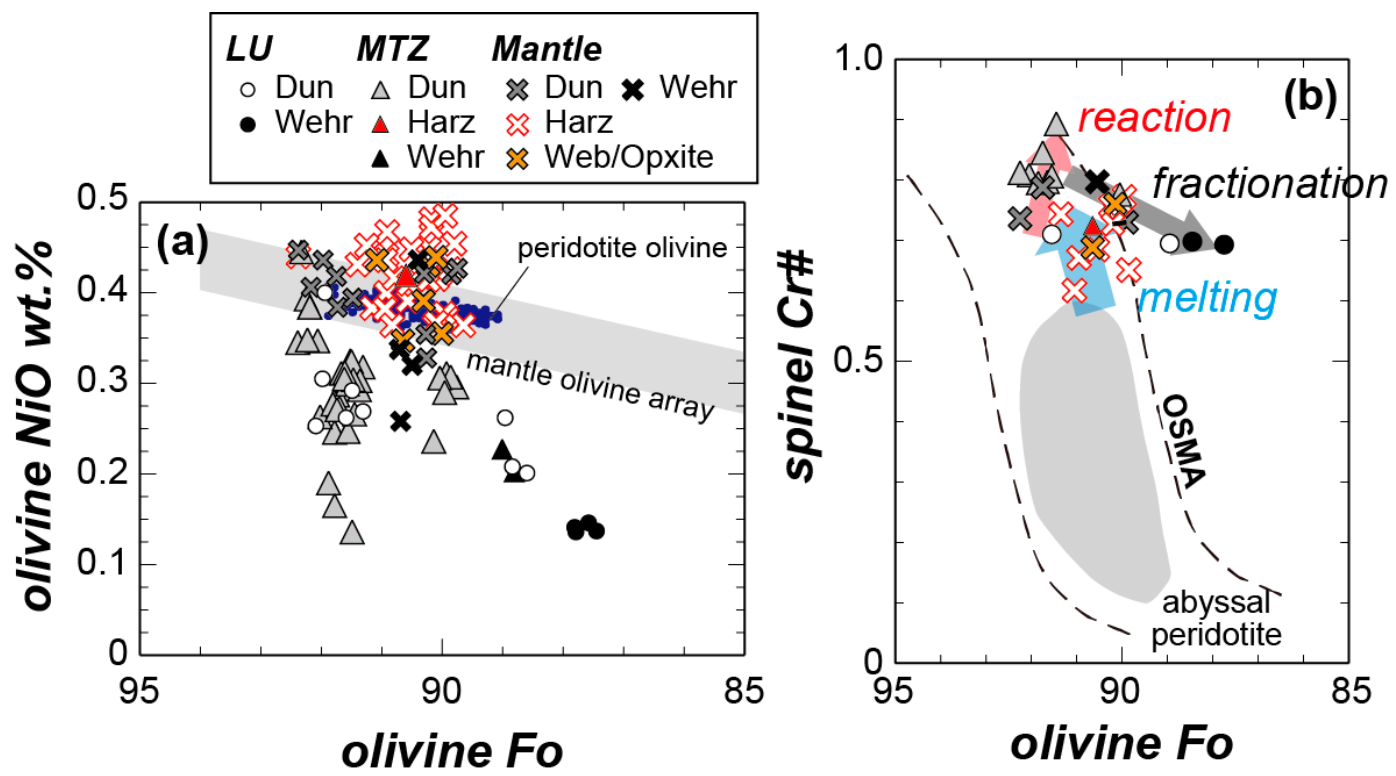

Figure 7. (a) Relationships between Fo content and NiO content of olivine. Mantle olivine array [38] and mantle peridotite olivine (blue) [39] show the compositional variation of residual mantle peridotite. (b) Chemical variations between Fo content of olivine and Cr\# of chromian spinel. Abyssal peridotite is shown for comparison [40]. OSMA shows the residual trend of spinel peridotite [40]. Tie-lined plots of mantle harzburgite and dunite is the sample from the boundary (2014MR2-09) (Tables 1-3). Arrows are showing evolution trends of the Mersin ultramafic rocks, of melting (blue), reaction (red), and fractionation (black), respectively, which are suggested by mineral chemistry (see discussion).

\subsubsection{MTZ Peridotites (Dunite-Wehrlite-Harzburgite of R1)}

Olivine composition of MTZ dunite is almost constant: Mg\# and NiO content are 0.91-0.92 and 0.28-0.31 wt.\%, respectively, although there are some exceptions (Figure 7a). Chromian spinel in MTZ dunite shows constant and high $\mathrm{Cr} \#(0.78-0.89)$ and low $\mathrm{TiO}_{2}$ content $(0.08-0.17 \mathrm{wt} . \%)$, and the $\mathrm{Mg \#}$ is slightly varied (0.38-0.44) (Figure 8). The Cr\# of chromian spinel in MTZ dunite is slightly higher than in LU rocks, and is similar to that in boninite (Figure 8). The Mg\# of clinopyroxene in MTZ dunite is high and constant (0.95-0.96), and their $\mathrm{TiO}_{2}, \mathrm{Al}_{2} \mathrm{O}_{3}$, and $\mathrm{Cr}_{2} \mathrm{O}_{3}$ contents are low: 0.01-0.05 wt.\%, 0.28-0.79 wt.\% and 0.05-0.61 wt.\%, respectively (Figure 10; Table 5). Amphibole inclusions in chromian spinel of MTZ dunite are pargasite with low $\mathrm{TiO}_{2}$ content (<0.26 wt.\%) and the $\mathrm{Mg \#}$ is 0.93 . 


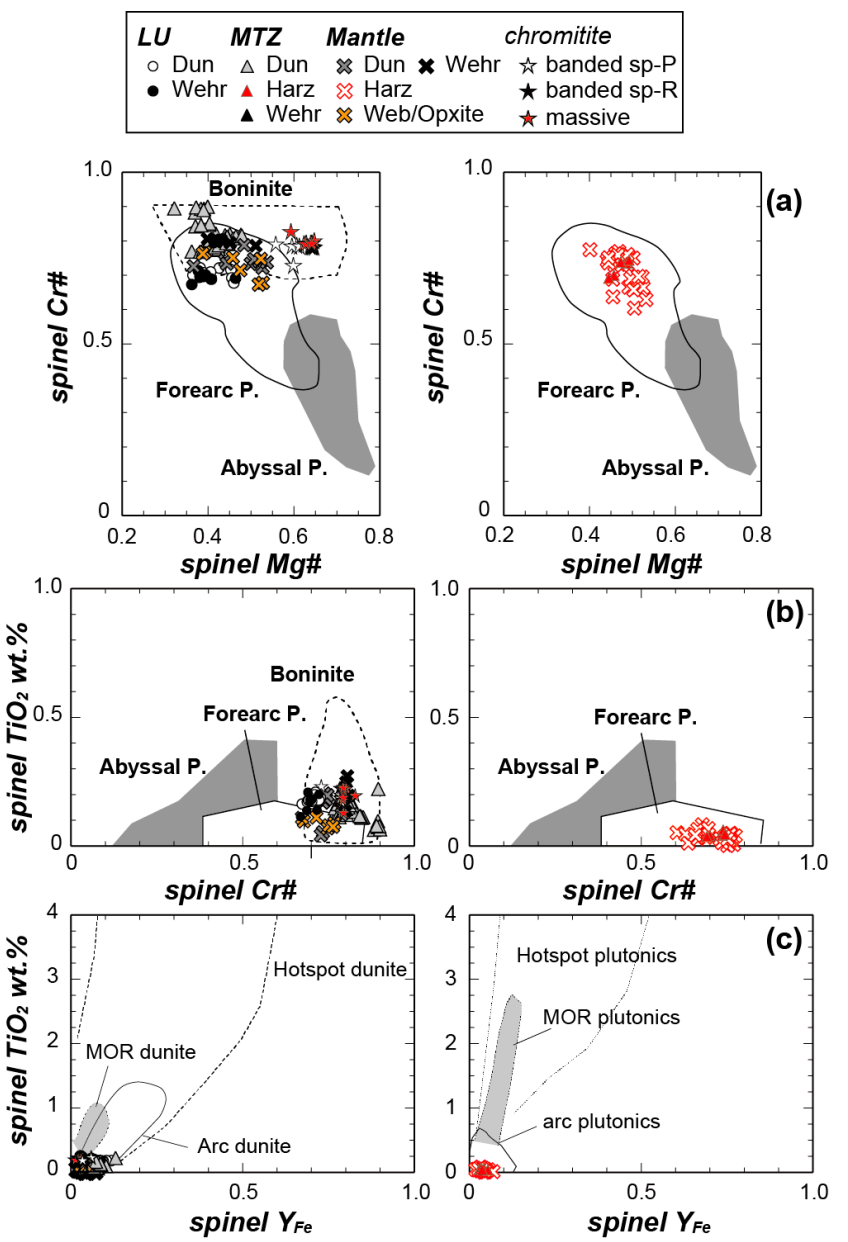

Figure 8. Chemical variations of chromian spinel in each rock type of the Mersin ultramafic rocks. Left and right panels are for dunite, chromitite, wehrlite, and websterite/orthopyroxenite, and for harzburgite, respectively. Data of banded chromitite is shown separately as spinel-rich part (graded sp-R) and spinel-poor part (graded sp-P). The fields for other key rocks, i.e., forearc P. (=peridotite), abyssal P., and boninite are shown for comparison, and original data sources are listed in Tamura and Arai [41]. (a) Relationships between Mg\# and Cr\# of chromian spinel. (b) Relationships between Cr\# and $\mathrm{TiO}_{2}$ content of chromian spinel. (c) Relationships between $\mathrm{Y}_{\mathrm{Fe}}$ and $\mathrm{TiO}_{2}$ content of chromian spinel. Fields for hotspot, MOR and arc rocks are from Arai et al. [42].

The $\mathrm{Mg} \#, \mathrm{Cr} \#$ and $\mathrm{Y}_{\mathrm{Fe}}$ of chromian spinel in massive chromitite (14T-MR01) are almost constant, $0.62-0.64, \approx 0.79$ and $0.013-0.036$, respectively, except for the rim or small grain which is surrounded by chlorite (Table 2; Figure 8). Clinopyroxene inclusions in chromian spinel of massive chromitite show high $\mathrm{Mg \#} \mathrm{(up} \mathrm{to} \mathrm{0.97)} \mathrm{and} \mathrm{almost} \mathrm{the} \mathrm{same} \mathrm{Al}_{2} \mathrm{O}_{3}(0.54 \mathrm{wt} . \%)$ as those in discrete grain in dunite, while the $\mathrm{Na}_{2} \mathrm{O}$ content is higher $(0.22 \mathrm{wt} . \%)$. Pargasite inclusions in chromitite of massive chromitite show almost the same $\mathrm{TiO}_{2}(0.24-0.39 \mathrm{wt} . \%)$ as those in spinel inclusion in MTZ dunite, but the Mg\# is quite high $0.95-0.96$ due to subsolidus $\mathrm{Mg}-\mathrm{Fe}^{2+}$ redistribution [43].

Olivine shows lower Mg\# (0.91) and higher NiO content (0.42 wt.\%) in MTZ harzburgite than in MTZ dunite (Table 2; Figure 7). Coexisting chromian spinel shows slightly lower Cr\# (0.69-74) and $\mathrm{TiO}_{2}$ content (0.04-0.06 wt.\%), and higher Mg\# (0.44-0.49) than in MTZ dunite (Table 3). The chromian spinel in MTZ harzburgite is similar in chemistry to that in forearc peridotite/arc plutonics (Figure 8c). Clinopyroxene in MTZ harzburgite shows lower Mg\# (0.93-0.95) and higher $\mathrm{Al}_{2} \mathrm{O}_{3}(0.77-1.15$ wt.\%) and $\mathrm{Cr}_{2} \mathrm{O}_{3}(0.35-0.73$ wt.\%) contents than in MTZ dunite (Table 5; Figure 10). Orthopyroxene in MTZ harzburgite shows almost the same $\mathrm{Mg} \#$ as olivine $(\approx 0.91)$ and the $\mathrm{Al}_{2} \mathrm{O}_{3}, \mathrm{Cr}_{2} \mathrm{O}_{3}$, and CaO contents are 0.82-1.10 wt.\%, 0.34-0.52 wt.\%, and 0.33-1.50 wt.\%, respectively (Table 4; Figure 9). 

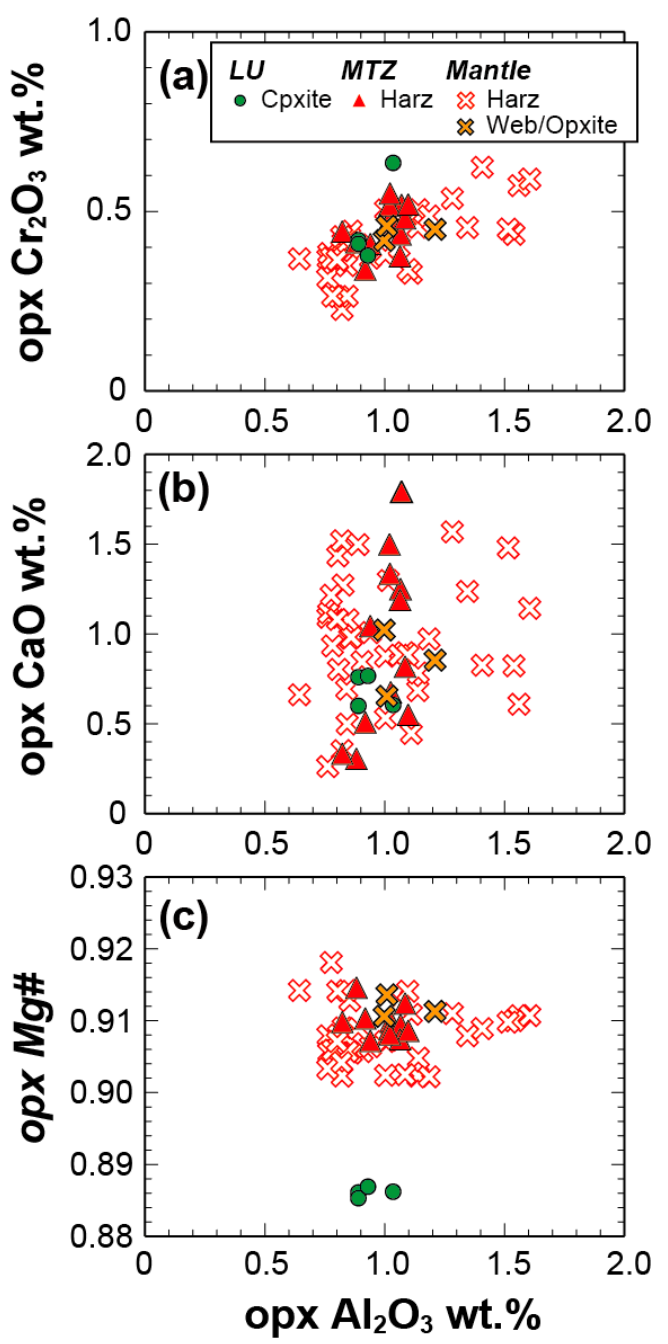

Figure 9. Chemical variations of orthopyroxenes in each rock type from the Mersin ultramafic rocks. (a) Relationships between $\mathrm{Al}_{2} \mathrm{O}_{3}$ wt.\% and $\mathrm{Cr}_{2} \mathrm{O}_{3}$ wt.\%. (b) Relationships between $\mathrm{Al}_{2} \mathrm{O}_{3}$ wt. $\%$ and $\mathrm{CaO}$ wt.\%. (c) Relationships between $\mathrm{Al}_{2} \mathrm{O}_{3}$ wt.\% and $\mathrm{Mg \#}$.

\subsubsection{Mantle Peridotites (Ultramafic Rocks of R2)}

Olivine in the mantle harzburgite show constant Mg\# (0.90-0.91) and slightly varied NiO contents (0.36-0.49 wt.\%) (Figure 7; Table 2). The Mg\# and Cr\# of chromian spinel in mantle harzburgite are high and varied, $0.40-0.53$ and $0.60-0.77$, respectively, but there is no obvious correlation between those values and modal amounts of pyroxenes (cf. Table 3 and Figure 8). The $\mathrm{TiO}_{2}$ content and $\mathrm{Y}_{\mathrm{Fe}}$ of the chromian spinel are low, $<0.01-0.07 \mathrm{wt} . \%$ and 0.02-0.07, respectively (Table 3; Figure 8b,c). The mantle harzburgite of R2 is similar to forearc peridotites in terms of spinel chemistry (Figure 8), but is slightly off the olivine-spinel mantle array (=OSMA) [40] to the low Fo content (olivine) area (Figure $7 \mathrm{~b}$ ). The $\mathrm{Mg \#}$ of clinopyroxene in mantle harzburgite is high and constant (0.93-0.95), and the $\mathrm{TiO}_{2}(<0.03 \mathrm{wt} . \%), \mathrm{Al}_{2} \mathrm{O}_{3}(0.62-1.72 \mathrm{wt} . \%)$, and $\mathrm{Cr}_{2} \mathrm{O}_{3}(0.28-0.88 \mathrm{wt} . \%)$ contents are higher than in MTZ harzburgite (Table 5; Figure 10). Orthopyroxene in mantle harzburgite shows almost the same Mg\# as olivine (0.90-0.91) and their $\mathrm{Al}_{2} \mathrm{O}_{3}, \mathrm{Cr}_{2} \mathrm{O}_{3}$, and $\mathrm{CaO}$ contents are, 0.64-1.41 wt.\%, 0.23-0.62 wt.\%, and $0.26-1.57$ wt.\%, respectively (Table 4; Figure 9). 

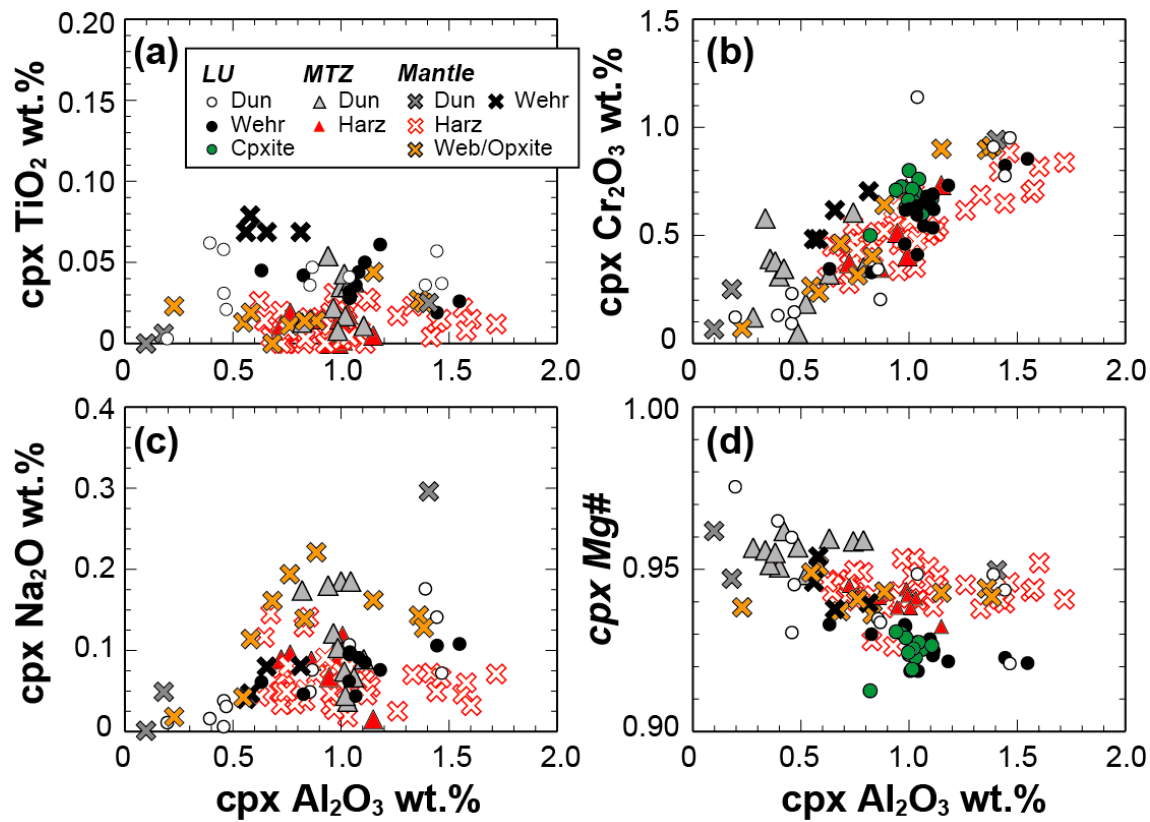

Figure 10. Chemical variations of clinopyroxenes in each rock type from the Mersin ultramafic rocks. (a) Relationships between $\mathrm{Al}_{2} \mathrm{O}_{3}$ wt. $\%$ and $\mathrm{TiO}_{2}$ wt.\%. (b) Relationships between $\mathrm{Al}_{2} \mathrm{O}_{3}$ wt.\% and $\mathrm{Cr}_{2} \mathrm{O}_{3}$ wt.\%. (c) Relationships between $\mathrm{Al}_{2} \mathrm{O}_{3}$ wt.\% and $\mathrm{Na}_{2} \mathrm{O}$ wt.\%. (d) Relationships between $\mathrm{Al}_{2} \mathrm{O}_{3}$ wt. $\%$ and $\mathrm{Mg \# .}$

The Mg\# of olivine in mantle dunite-clinopyroxenite-websterite-wehrlite is varied from 0.90-0.92 and the $\mathrm{NiO}$ content is mostly $0.30-0.43 \mathrm{wt} . \%$, irrespective of the rock species (Table 2; Figure 7). Chromian spinel in mantle dunite-clinopyroxenite-websterite-wehrlite also shows constant chemical compositions and are plotted in the transitional area between those of boninite and forearc peridotites or arc cumulative rocks: The $\mathrm{TiO}_{2}$ content, $\mathrm{Mg} \#$ and $\mathrm{Cr} \#$ are $0.04-0.26$ wt. $\%, 0.37-0.54$ and $0.72-0.81$, respectively, although the spinel in websterite (2013MR5-02) shows slightly low Cr\# (0.69) (Table 3; Figure 8). The $\mathrm{Mg} \#$ of clinopyroxene in mantle dunite-clinopyroxenite-websterite is high and constant (0.94-0.96), and the variations of $\mathrm{TiO}_{2}, \mathrm{Al}_{2} \mathrm{O}_{3}$, and $\mathrm{Cr}_{2} \mathrm{O}_{3}$ contents of clinopyroxene are almost the same as in the MTZ dunite, although $\mathrm{Na}_{2} \mathrm{O}$ content (up to $0.30 \mathrm{wt} . \%$ ) is slightly higher than in the MTZ dunite: $<0.08 \mathrm{wt} . \%, 0.10-1.41 \mathrm{wt} . \%$, and 0.07-0.94, respectively (Table 5; Figure 10). Orthopyroxene in websterite shows almost the same $\mathrm{Mg} \#$ as olivine (0.91), and the $\mathrm{Al}_{2} \mathrm{O}_{3}, \mathrm{Cr}_{2} \mathrm{O}_{3}$, and $\mathrm{CaO}$ contents are 1.00-1.21 wt.\%, 0.42-0.45 wt.\%, and 0.65-1.02 wt.\%, respectively (Table 4; Figure 9). Amphibole inclusions in some chromian spinel grains in dunite and websterite are varied from edenite to tremolite with low $\mathrm{TiO}_{2}$ contents $(0.09-0.27$ wt. $\%)$.

The $\mathrm{Cr} \#$ and $\mathrm{Y}_{\mathrm{Fe}}$ of chromian spinel in banded chromitite, showing graded bedding, are almost constant, $0.78-0.79$ and $0.03-0.04$, respectively, while $\mathrm{Mg} \#$ is slightly higher $(0.64-0.65)$ in spinel-rich zone than in spinel-poor zone (0.56-0.61) (Figure 8; Table 3), due to subsolidus $\mathrm{Mg}-\mathrm{Fe}^{2+}$ redistribution [43]. Olivine inclusion in chromian spinel shows higher $\mathrm{Mg \#}$ and $\mathrm{NiO}$ content $(\approx 0.96$ and $0.53-0.58 \mathrm{wt} . \%$ ) than discrete olivine grains (0.91 and $0.28 \mathrm{wt} . \%$, respectively), which is also due to subsolidus ${\mathrm{Mg}-\mathrm{Fe}^{2+}}^{2+}$ redistribution. Clinopyroxene inclusions in chromian spinel show high $\mathrm{Mg \#} \mathrm{(0.96)}$ and almost the same $\mathrm{Al}_{2} \mathrm{O}_{3}$ and $\mathrm{Cr}_{2} \mathrm{O}_{3}$ content $(0.79 \mathrm{wt} . \%$ and $0.95 \mathrm{wt} . \%)$ with those in discrete grain in dunite, wehrlite, and websterite/orthopyroxenite. $\mathrm{TiO}_{2}$ content, $\mathrm{Mg}$, and $\mathrm{Na} /(\mathrm{Na}+\mathrm{K})$ atomic ratio of phlogopite are $\approx 0.35 \mathrm{wt} . \%, 0.97$ and $\approx 0.11$, respectively. There is no large difference in mineral chemistry between massive chromitite (14T-MR01) and banded chromitite (14T-MR12), except for Mg\# of minerals. 


\subsection{Trace-Element Compositions}

We determined trace-element compositions of clinopyroxene for each sample and orthopyroxene, if any, i.e., harzburgite and pyroxenites, and the results are shown in Tables 6 and 7 and Figures 11 and 12.
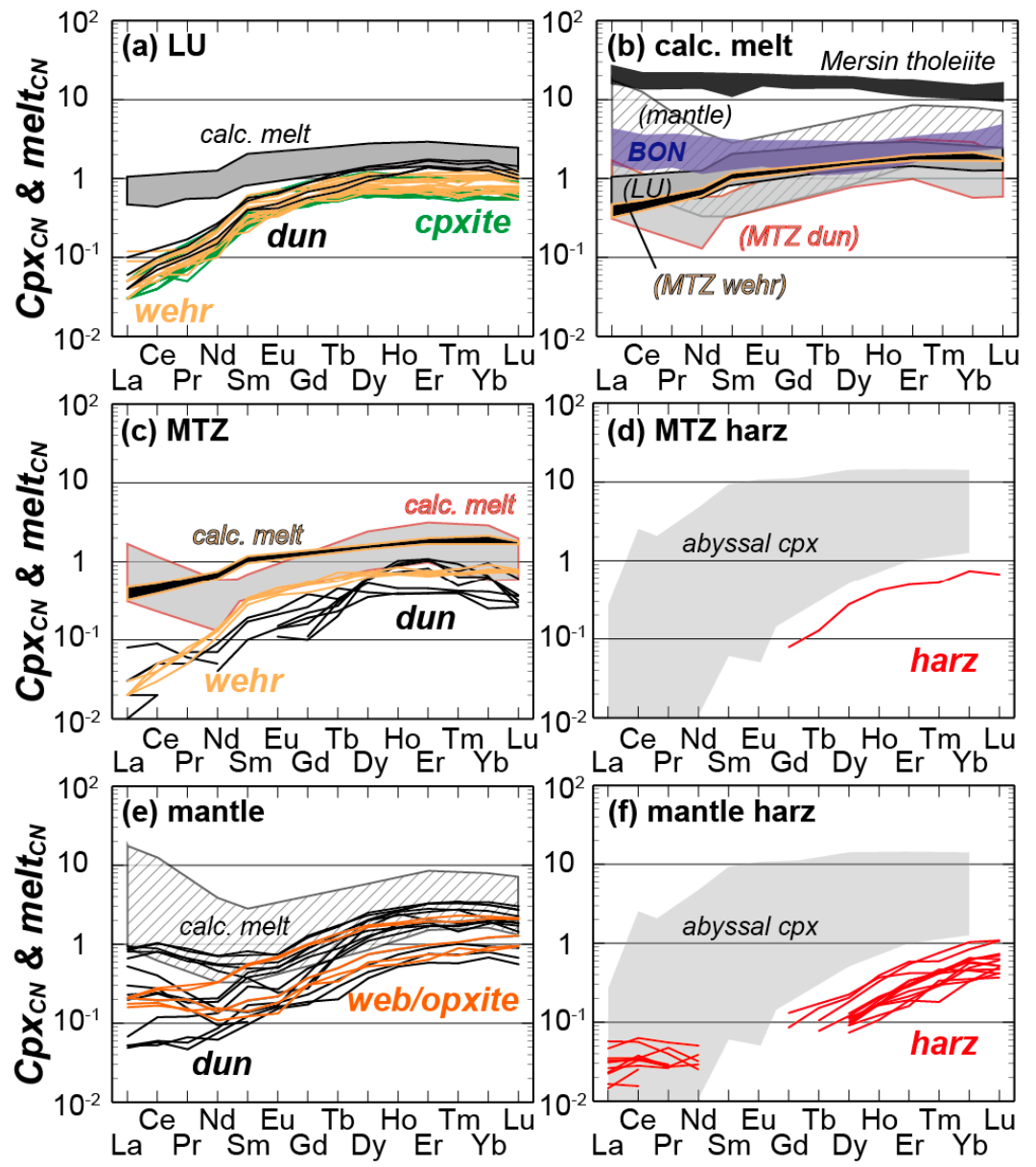

Figure 11. REE patterns, which are normalized to chondrite values [44], of clinopyroxene and the calculated melt in equilibrium with clinopyroxene (calc. melt). Abyssal peridotites [45-47] in panel $(\mathbf{d}, \mathbf{f})$ are for reference. Data vacancy means abundances below the detection limits (see Table 6). (a) LU dunite (black), wehrlite (orange) and clinopyroxenite (green). Calculated melt in equilibrium with clinopyroxene (gray field) in all rocks are almost the same in pattern and shown in gray color all together. (b) Calculated melt in equilibrium with clinopyroxene in dunite, wehrlite, clinopyroxenite, and websterite/orthopyroxenite is shown for each section. Patterns of the Mersin tholeiitic basalt (black) [9] and boninite (BON) from Izu-Ogasawara arc (violet) [48] are for reference. (c) MTZ dunite (black) and wehrlite (orange). Calculated melts in equilibrium with clinopyroxene in wehrlite and dunite are black field outlined by orange and gray field outlined by red, respectively. (d) MTZ harzburgite. (e) Mantle dunite (black) and websterite/orthopyroxenite (dark orange). Calculated melt in equilibrium with clinopyroxene in websterite/orthopyroxenite is within the range of those in dunite and not specifically shown (field filled by oblique stripe). (f) Mantle harzburgite. 

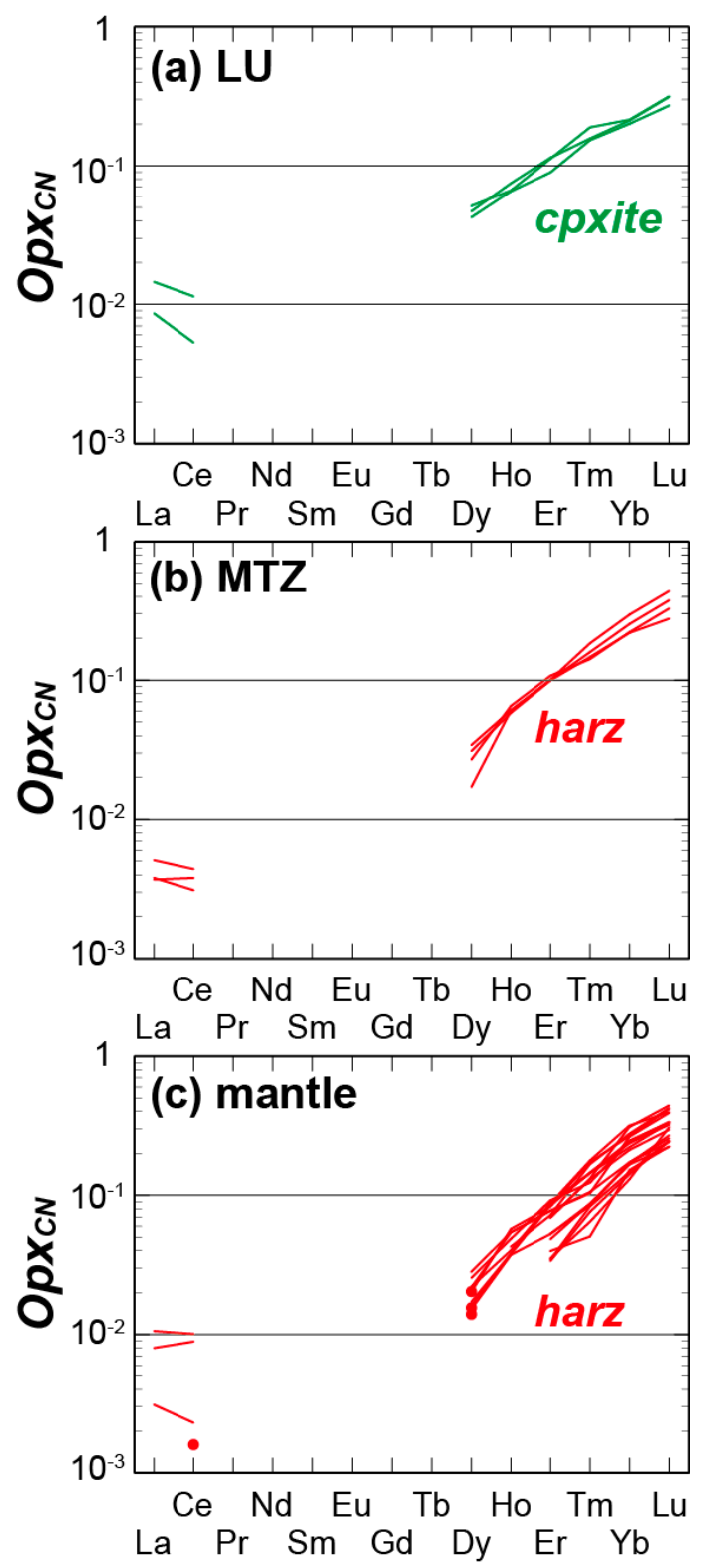

Figure 12. REE patterns, which are normalized to chondrite values [44], of orthopyroxene in the Mersin ultramafic rocks. No data means below their detection limits (Table 7). (a) Clinopyroxenite of LU. (b) MTZ harzburgite. (c) Mantle harzburgite.

\subsubsection{LU Rocks (Dunite-Wehrlite-Clinopyroxenite of R1)}

Rare earth element (REE) patterns, normalized to chondrite values [44] (subscript CN hereafter), of clinopyroxenes in LU rocks show gently light REE (LREE) depleted shape and their abundances are almost constant irrespective of host rock lithology (dunite, wehrlite, and clinopyroxenite) (Figure 11a). There is a weak correlation between clinopyroxene $\mathrm{REE}_{\mathrm{CN}}$ abundances and the stratigraphical position of host rock; the $\mathrm{REE}_{\mathrm{CN}}$ of clinopyroxene roughly decreases down-section (Table 6). Other incompatible elements are mostly below their detection limits in abundance, except for $\mathrm{Nb}, \mathrm{Pb}, \mathrm{Sr}$, and $\mathrm{Zr}$, and there is no systematic increase or decrease upsection or downsection (Table 6).

Chondrite normalized REE patterns of orthopyroxene in clinopyroxenite of the LU rocks show slightly steeper slope from Lu to Dy than those of coexisting clinopyroxene and most of LREE middle REE (MREE), except for La and Ce, are below their detection limits in abundance (Figure 12). Most of 
other incompatible trace elements were not detected, and if detected, the abundances are almost the same as or lower than in coexisting clinopyroxene (Table 7).

\subsubsection{MTZ Peridotites (Dunite-Wehrlite-Harzburgite of R1)}

Abundances of some REE in clinopyroxenes in MTZ peridotites are below their detection limits and almost the same as those in the LU rocks (Figure 11a,c). REE patterns of clinopyroxene in MTZ wehrlite are almost constant and show the same shape and abundances as in LU wehrlite (Figure 11a,c). Clinopyroxenes in some MTZ dunite samples show slightly different $R_{E E}$ patterns with a hump at MREE to heavy REE (HREE) and a peak at Ho to Er (Figure 11c). REE patterns of clinopyroxenes show slightly steeper slopes from HREE to MREE in MTZ harzburgite than in MTZ dunite (Figure 11c,d), although the abundances are almost the same. Other incompatible elements are mostly below their detection limits except for $\mathrm{Ba}, \mathrm{Nb}, \mathrm{Pb}, \mathrm{Sr}$, and $\mathrm{Zr}$ as those in the $\mathrm{LU}$ rocks (Table 6).

$\mathrm{REE}_{\mathrm{CN}}$ patterns of orthopyroxene in MTZ harzburgite show steeper slope from Lu to Dy and lower abundances than those of coexisting clinopyroxene (Figure 12). Most of other incompatible trace elements are below their detection limits, and if detected, the abundances are almost the same as or lower than coexisting clinopyroxene as in clinopyroxenite of the LU rocks (Table 7). The concentrations of some large ion lithophile elements (LILE: $\mathrm{Rb}, \mathrm{Ba}$, and $\mathrm{Sr}$ ) in these orthopyroxenes are slightly higher than those of mantle harzburgite, as shown in the following Section 4.3.3 (Table 7).

\subsubsection{Mantle Peridotites (Ultramafic Rocks of R2)}

$\mathrm{REE}_{\mathrm{CN}}$ patterns of clinopyroxenes in mantle dunite are highly varied and roughly divided into two types: One shows simple gentle slope from HREE to LREE and the other, LREE-enriched spoon shaped pattern (Figure 11e). The shape of the former, as well as abundances, is almost the same as those in LU rocks and MTZ wehrlite (Figure 11a,c). On the other hand, the latter shows higher $\mathrm{REE}_{\mathrm{CN}}$ abundances and some show some flattened patterns (Figure 11e). The $\mathrm{REE}_{\mathrm{CN}}$ patterns of clinopyroxene in mantle websterite/orthopyroxenite are within the variation of those in dunite (Figure 11e).

$\mathrm{REE}_{\mathrm{CN}}$ pattern of clinopyroxenes in mantle harzburgite is almost the same, although the LREE abundance is slightly higher, as that in MTZ harzburgite (Figure 11d,f). The $\operatorname{REE}_{\mathrm{CN}}$ abundance of these clinopyroxenes is obviously low relative to those of abyssal peridotites (Figure 11e). There is no clear correlation between the REE abundance of clinopyroxenes and the stratigraphical position of host rock (Table 6). Other incompatible elements are mostly below their detection limits except $\mathrm{Nb}, \mathrm{Pb}$, $\mathrm{Sr}$, and $\mathrm{Zr}$ as those in the rocks of MTZ harzburgite (Table 6).

$\mathrm{REE}_{\mathrm{CN}}$ patterns of orthopyroxene in mantle harzburgite show almost the same as those in MTZ harzburgite, although the abundances are slightly lower in the former (Figure 12). Most of other incompatible trace elements are below their detection limits, and if detected, the abundances are almost the same or lower than coexisting clinopyroxene as in other samples (Table 7). 
Table 6. Representative trace-element compositions of clinopyroxene in the Mersin ultramafic rocks.

\begin{tabular}{|c|c|c|c|c|c|c|c|c|c|c|c|c|c|c|c|}
\hline \multirow{3}{*}{$\begin{array}{l}\text { Rock Suite } \\
\text { Rock Type } \\
\text { Sample No. }\end{array}$} & \multicolumn{8}{|l|}{ LU Rocks } & \multicolumn{7}{|l|}{ MTZ Rocks } \\
\hline & Dunite & Wehrlite & & & & Cpxite & & & Dunite & & & & & Wehrlite & Harz \\
\hline & 2013MR2-0 & 2013MR3-02 & 2013MR2-02 & 2013MR2-06 & 2013MR2-09 & 2013MR3-01 & 2013MR2-08 & 2013MR2-10 & 2014MR1-01 & 2013-MR1-03 & 3 2014MR1-06 & 2014MR1-09 & 2014MR1-13 & 2014MR1-16 & 2013MR1-02 \\
\hline $\begin{array}{c}\text { No. of } \\
\text { Analysis }\end{array}$ & $(\mathrm{N}=4)$ & $(\mathrm{N}=4)$ & $(\mathrm{N}=5)$ & $(\mathrm{N}=4)$ & $(\mathrm{N}=6)$ & $(\mathrm{N}=3)$ & $(N=6)$ & $(\mathrm{N}=4)$ & $(\mathrm{N}=3)$ & $(\mathrm{N}=1)$ & $(\mathrm{N}=3)$ & $(\mathrm{N}=1)$ & $(\mathrm{N}=2)$ & $(\mathrm{N}=5)$ & $(\mathrm{N}=1)$ \\
\hline $\mathrm{Rb}$ & $(<0.040)$ & $(<0.040)$ & $(<0.040)$ & $(<0.040)$ & 0.072 & $(<0.040)$ & 0.047 & $(<0.040)$ & 0.096 & $(<0.040)$ & $(<0.040)$ & $(<0.040)$ & $(<0.040)$ & $(<0.040)$ & $(<0.040)$ \\
\hline $\mathrm{Sr}$ & 3.751 & 3.157 & 3.872 & 2.674 & 2.53 & 3.646 & 2.413 & 2.171 & 1.049 & 0.457 & 1.662 & 0.627 & 1.988 & 2.499 & 4.307 \\
\hline $\mathrm{Y}$ & 1.95 & 1.319 & 1.338 & 0.959 & 0.948 & 1.453 & 0.886 & 0.832 & 1.191 & 1.725 & 0.576 & 0.862 & 1.313 & 0.946 & 0.555 \\
\hline $\mathrm{Zr}$ & 0.449 & 0.203 & 0.249 & 0.27 & 0.132 & 0.231 & 0.103 & 0.114 & 0.282 & 0.2 & 0.089 & 0.162 & 0.21 & 0.1 & 0.053 \\
\hline $\mathrm{Nb}$ & 0.045 & 0.037 & 0.043 & 0.042 & 0.034 & 0.019 & 0.032 & 0.036 & 0.021 & 0.022 & 0.023 & 0.022 & 0.035 & 0.033 & 0.021 \\
\hline Cs & $(<0.023)$ & $(<0.023)$ & $(<0.023)$ & $(<0.023)$ & $(<0.023)$ & $(<0.023)$ & $(<0.023)$ & $(<0.023)$ & $(<0.023)$ & $(<0.023)$ & $(<0.023)$ & $(<0.023)$ & $(<0.023)$ & $(<0.023)$ & $(<0.023)$ \\
\hline Ва & 0.258 & 0.05 & 0.286 & 0.246 & 0.242 & 0.059 & 0.111 & 0.096 & 0.155 & 0.086 & 0.078 & $(<0.045)$ & 0.108 & 0.108 & 0.349 \\
\hline $\mathrm{Ce}$ & 0.057 & 0.051 & 0.056 & 0.036 & 0.037 & 0.055 & 0.029 & 0.031 & 0.026 & 0.013 & 0.023 & 0.028 & 0.053 & 0.026 & 0.002 \\
\hline $\operatorname{Pr}$ & 0.012 & 0.011 & 0.011 & 0.007 & 0.008 & 0.013 & 0.007 & 0.006 & 0.003 & 0.002 & 0.004 & 0.005 & 0.007 & 0.006 & $(<0.002)$ \\
\hline $\mathrm{Nd}$ & 0.097 & 0.091 & 0.091 & 0.064 & 0.064 & 0.104 & 0.057 & 0.059 & 0.016 & 0.014 & 0.031 & 0.035 & 0.045 & 0.06 & $(<0.011)$ \\
\hline $\mathrm{Sm}$ & 0.07 & 0.074 & 0.063 & 0.046 & 0.048 & 0.081 & 0.045 & 0.044 & $(<0.011)$ & 0.018 & 0.022 & 0.03 & 0.028 & 0.047 & $(<0.011)$ \\
\hline $\mathrm{Eu}$ & 0.031 & 0.033 & 0.029 & 0.021 & 0.023 & 0.04 & 0.022 & 0.021 & 0.008 & 0.01 & 0.011 & 0.012 & 0.015 & 0.023 & $(<0.006)$ \\
\hline $\mathrm{Gd}$ & 0.15 & 0.14 & 0.147 & 0.11 & 0.107 & 0.157 & 0.098 & 0.093 & 0.031 & 0.089 & 0.056 & 0.067 & 0.091 & 0.1 & 0.017 \\
\hline $\mathrm{Tb}$ & 0.034 & 0.03 & 0.03 & 0.023 & 0.023 & 0.034 & 0.022 & 0.019 & 0.009 & 0.028 & 0.012 & 0.015 & 0.023 & 0.022 & 0.005 \\
\hline Dy & 0.297 & 0.235 & 0.235 & 0.18 & 0.172 & 0.276 & 0.165 & 0.163 & 0.152 & 0.265 & 0.104 & 0.151 & 0.238 & 0.174 & 0.069 \\
\hline Ho & 0.072 & 0.052 & 0.053 & 0.038 & 0.038 & 0.058 & 0.036 & 0.033 & 0.049 & 0.073 & 0.022 & 0.031 & 0.055 & 0.04 & 0.023 \\
\hline Er & 0.248 & 0.155 & 0.155 & 0.113 & 0.117 & 0.17 & 0.103 & 0.1 & 0.16 & 0.195 & 0.065 & 0.106 & 0.139 & 0.115 & 0.08 \\
\hline $\mathrm{Tm}$ & 0.036 & 0.023 & 0.025 & 0.016 & 0.016 & 0.023 & 0.015 & 0.016 & 0.017 & 0.033 & 0.01 & 0.012 & 0.019 & 0.018 & 0.013 \\
\hline $\mathrm{Yb}$ & 0.24 & 0.156 & 0.176 & 0.11 & 0.123 & 0.175 & 0.109 & 0.095 & 0.103 & 0.201 & 0.053 & 0.076 & 0.11 & 0.128 & 0.121 \\
\hline $\mathrm{Lu}$ & 0.029 & 0.024 & 0.027 & 0.016 & 0.018 & 0.025 & 0.015 & 0.014 & 0.008 & 0.021 & 0.008 & 0.009 & 0.015 & 0.018 & 0.017 \\
\hline $\mathrm{Pb}$ & 0.119 & 0.061 & 0.594 & 0.181 & 0.201 & 0.051 & 0.164 & 0.189 & 0.648 & 0.126 & 0.068 & 0.113 & $(<0.035)$ & 0.093 & 0.106 \\
\hline Th & $(<0.006)$ & $(<0.006)$ & $(<0.006)$ & $(<0.006)$ & $(<0.006)$ & $(<0.006)$ & $(<0.006)$ & $(<0.006)$ & $(<0.006)$ & $(<0.006)$ & $(<0.006)$ & $(<0.006)$ & $(<0.006)$ & $(<0.006)$ & $(<0.006)$ \\
\hline $\mathrm{U}$ & $(<0.005)$ & $(<0.005)$ & 0.013 & 0.016 & 0.011 & $(<0.005)$ & $(<0.005)$ & $(<0.005)$ & 0.008 & $(<0.005)$ & $(<0.005)$ & $(<0.005)$ & 0.006 & $(<0.005)$ & $(<0.005)$ \\
\hline Rock Suite & Mantle Rocks & & & & & & & & & & & & & & \\
\hline Rock Type & Harz & & & & & & & Dunite & & $\begin{array}{l}\text { (Dunite } \\
\text { part) }\end{array}$ & Dunite & $\begin{array}{l}\text { (Dunite } \\
\text { part) }\end{array}$ & Dunite & Websterite & (Web part) \\
\hline Sample No. & 2014MR2-16 & 2014MR2-12 & 2013MR7-01 & 2014MR2-03 & 2014MR2-01 & 2013MR4-04 & 2013MR8-01 & 2014MR2-14 & 2014MR2-11 & 2014MR2-07 & 2013MR5-01 & 2013MR4-05 & 2013MR4-03 & 2014MR2-10 & 2014MR2-07 \\
\hline $\begin{array}{c}\text { No. of } \\
\text { Analysis }\end{array}$ & $(\mathrm{N}=2)$ & $(\mathrm{N}=2)$ & $(\mathrm{N}=4)$ & $(\mathrm{N}=2)$ & $(\mathrm{N}=1)$ & $(\mathrm{N}=1)$ & $(\mathrm{N}=3)$ & $(\mathrm{N}=3)$ & $(\mathrm{N}=3)$ & $(\mathrm{N}=1)$ & $(\mathrm{N}=1)$ & $(\mathrm{N}=2)$ & $(\mathrm{N}=3)$ & $(\mathrm{N}=4)$ & $(\mathrm{N}=2)$ \\
\hline $\mathrm{Rb}$ & $(<0.040)$ & $(<0.040)$ & $(<0.040)$ & $(<0.040)$ & $(<0.040)$ & $(<0.040)$ & $(<0.040)$ & 0.679 & $(<0.040)$ & 0.049 & $(<0.040)$ & $(<0.040)$ & $(<0.040)$ & $(<0.040)$ & $(<0.040)$ \\
\hline $\mathrm{Sr}$ & 1.225 & 1.273 & 1.123 & 0.972 & 1.517 & 1.81 & 0.637 & 9.896 & 25.548 & 5.184 & 17.423 & 7.334 & 3.413 & 8.971 & 8.09 \\
\hline $\mathrm{Y}$ & 0.156 & 0.438 & 0.2 & 0.1 & 0.549 & 0.515 & 0.466 & 2.811 & 3.84 & 1.935 & 3.57 & 2.192 & 0.86 & 1.033 & 2.523 \\
\hline $\mathrm{Zr}$ & 0.083 & 0.055 & 0.045 & 0.045 & 0.043 & 0.102 & 0.053 & 2.979 & 1.722 & 0.328 & 1.486 & 0.551 & 0.084 & 0.315 & 0.522 \\
\hline $\mathrm{Nb}$ & 0.03 & 0.036 & 0.035 & 0.031 & 0.03 & 0.036 & 0.027 & 0.247 & 0.062 & 0.04 & 0.056 & 0.045 & 0.015 & 0.039 & 0.058 \\
\hline Cs & $(<0.023)$ & $(<0.023)$ & $(<0.023)$ & $(<0.023)$ & $(<0.023)$ & $(<0.023)$ & $(<0.023)$ & 0.033 & $(<0.023)$ & $(<0.023)$ & $(<0.023)$ & $(<0.023)$ & $(<0.023)$ & $(<0.023)$ & $(<0.023)$ \\
\hline Ba & $(<0.045)$ & $(<0.045)$ & $(<0.045)$ & $(<0.045)$ & $(<0.045)$ & $(<0.045)$ & $(<0.045)$ & 0.613 & 0.109 & 0.04 & $(<0.045)$ & $(<0.045)$ & $(<0.045)$ & 0.079 & $(<0.045)$ \\
\hline $\mathrm{La}$ & 0.007 & 0.006 & 0.007 & 0.004 & 0.014 & 0.011 & $(<0.002)$ & 0.14 & 0. & 0.016 & 0.192 & 0.051 & 0.012 & 0.0 & 0.05 \\
\hline $\mathrm{Ce}$ & 0.023 & 0.019 & & 0.013 & & 0.04 & 0.006 & 0.319 & 0.589 & 0.073 & 0.4 & 0.148 & 0.035 & 0.114 & 0.167 \\
\hline $\operatorname{Pr}$ & 0.004 & 0.002 & $(<0.002)$ & $(<0.002)$ & 0.004 & 0.005 & $(<0.002)$ & 0.033 & 0.073 & 0.011 & 0.057 & 0.018 & 0.005 & 0.016 & 0.026 \\
\hline $\mathrm{Nd}$ & 0.017 & 0.011 & $(<0.011)$ & 0.014 & 0.012 & 0.024 & $(<0.011)$ & 0.107 & 0.303 & 0.062 & 0.248 & 0.09 & 0.034 & 0.062 & 0.152 \\
\hline $\mathrm{Sm}$ & $(<0.011)$ & $(<0.011)$ & $(<0.011)$ & $(<0.011)$ & $(<0.011)$ & $(<0.011)$ & $(<0.011)$ & 0.03 & 0.105 & 0.043 & 0.073 & 0.061 & 0.019 & 0.024 & 0.082 \\
\hline
\end{tabular}


Table 6. Cont.

\begin{tabular}{|c|c|c|c|c|c|c|c|c|c|c|c|c|c|c|c|}
\hline Rock Suite & Mantle Rocks & & & & & & & & & & & & & & \\
\hline Rock Type & Harz & & & & & & & Dunite & & $\begin{array}{l}\text { (Dunite } \\
\text { part) }\end{array}$ & Dunite & $\begin{array}{l}\text { (Dunite } \\
\text { part) }\end{array}$ & Dunite & Websterite & (Web part) \\
\hline Sample No. & 2014MR2-16 & 2014MR2-12 & 2013MR7-01 & 2014MR2-03 & 2014MR2-01 & 2013MR4-04 & 2013MR8-01 & 2014MR2-14 & 2014MR2-11 & 2014MR2-07 & 2013MR5-01 & 2013MR4-05 & 2013MR4-03 & 2014MR2-10 & 2014MR2-07 \\
\hline $\begin{array}{c}\text { No. of } \\
\text { Analysis }\end{array}$ & $(\mathrm{N}=2)$ & $(\mathrm{N}=2)$ & $(\mathrm{N}=4)$ & $(\mathrm{N}=2)$ & $(\mathrm{N}=1)$ & $(\mathrm{N}=1)$ & $(\mathrm{N}=3)$ & $(N=3)$ & $(\mathrm{N}=3)$ & $(\mathrm{N}=1)$ & $(\mathrm{N}=1)$ & $(\mathrm{N}=2)$ & $(\mathrm{N}=3)$ & $(\mathrm{N}=4)$ & $(\mathrm{N}=2)$ \\
\hline $\mathrm{Eu}$ & $(<0.006)$ & $(<0.006)$ & $(<0.006)$ & $(<0.006)$ & $(<0.006)$ & $(<0.006)$ & $(<0.006)$ & 0.009 & 0.039 & $(<0.006)$ & 0.028 & 0.027 & 0.009 & 0.011 & 0.039 \\
\hline $\mathrm{Gd}$ & $(<0.016)$ & $(<0.016)$ & $(<0.016)$ & 0.017 & 0.018 & 0.027 & $(<0.016)$ & 0.067 & 0.227 & 0.099 & 0.176 & 0.145 & 0.039 & 0.068 & 0.199 \\
\hline $\mathrm{Tb}$ & $(<0.004)$ & $(<0.004)$ & $(<0.004)$ & $(<0.004)$ & 0.006 & 0.006 & $(<0.004)$ & 0.03 & 0.057 & 0.025 & 0.049 & 0.037 & 0.01 & 0.016 & 0.046 \\
\hline Dy & 0.021 & 0.042 & 0.027 & 0.026 & 0.052 & 0.057 & 0.048 & 0.401 & 0.564 & 0.274 & 0.507 & 0.342 & 0.112 & 0.161 & 0.42 \\
\hline Ho & 0.008 & 0.016 & 0.01 & 0.008 & 0.022 & 0.021 & 0.017 & 0.123 & 0.145 & 0.075 & 0.145 & 0.089 & 0.033 & 0.04 & 0.1 \\
\hline $\mathrm{Er}$ & 0.033 & 0.084 & 0.053 & 0.029 & 0.096 & 0.069 & 0.072 & 0.359 & 0.491 & 0.271 & 0.527 & 0.29 & 0.12 & 0.134 & 0.329 \\
\hline $\mathrm{Yb}$ & 0.068 & 0.151 & 0.103 & 0.057 & 0.139 & 0.097 & 0.111 & 0.312 & 0.483 & 0.316 & 0.553 & 0.335 & 0.13 & 0.169 & 0.353 \\
\hline $\mathrm{Lu}$ & 0.013 & 0.027 & 0.018 & 0.011 & 0.027 & 0.013 & 0.017 & 0.048 & 0.064 & 0.035 & 0.075 & 0.046 & 0.018 & 0.028 & 0.052 \\
\hline $\mathrm{Hf}$ & $(<0.026)$ & $(<0.026)$ & $(<0.026)$ & $(<0.026)$ & $(<0.026)$ & $(<0.026)$ & $(<0.026)$ & 0.079 & 0.073 & 0.019 & 0.057 & $(<0.026)$ & $(<0.026)$ & $(<0.026)$ & $(<0.026)$ \\
\hline $\mathrm{Ta}$ & $(<0.007)$ & $(<0.007)$ & $(<0.007)$ & $(<0.007)$ & $(<0.007)$ & $(<0.007)$ & $(<0.007)$ & 0.018 & $(<0.007)$ & $(<0.007)$ & $(<0.007)$ & $(<0.007)$ & $(<0.007)$ & $(<0.007)$ & $(<0.007)$ \\
\hline $\mathrm{Pb}$ & 0.062 & 0.066 & 0.052 & 0.121 & 0.044 & 0.089 & 0.124 & 0.383 & 0.081 & 0.037 & 0.045 & 0.06 & 0.043 & 0.523 & 0.065 \\
\hline Th & $(<0.006)$ & $(<0.006)$ & $(<0.006)$ & $(<0.006)$ & $(<0.006)$ & $(<0.006)$ & $(<0.006)$ & 0.055 & $(<0.006)$ & $(<0.006)$ & $(<0.006)$ & $(<0.006)$ & $(<0.006)$ & $(<0.006)$ & 0.006 \\
\hline $\mathrm{U}$ & $(<0.005)$ & $(<0.005)$ & $(<0.005)$ & $(<0.005)$ & $(<0.005)$ & $(<0.005)$ & $(<0.005)$ & 0.048 & $(<0.005)$ & $(<0.005)$ & $(<0.005)$ & $(<0.005)$ & $(<0.005)$ & $(<0.005)$ & $(<0.005)$ \\
\hline
\end{tabular}

Italic values in parentheses are shown their detection limits when analyzed data below their detection limits. If there are different lithological parts within one sample, it is noted, e.g., (Harz part). Abbreviations of rock lithology are the same as Figure 2. 
Table 7. Representative trace-element compositions of orthopyroxene in the Mersin ultramafic rocks.

\begin{tabular}{|c|c|c|c|c|c|c|c|c|c|c|}
\hline Rock Suite & LU Rocks & & MTZ Rocks & & Mantle Rocks & & & & & \\
\hline Rock Type & Cpxite & & Harz & & Harz & & & & & \\
\hline Sample No. & 2013MR2-08 & 2013MR2-10 & 2013MR1-02 & 2014MR2-16 & 2014MR2-12 & 2013MR7-01 & 2014MR2-03 & 2014MR2-01 & 2013MR4-04 & 2013MR8-01 \\
\hline No. of Analysis & $(\mathrm{N}=1)$ & $(\mathrm{N}=1)$ & $(\mathrm{N}=4)$ & $(\mathrm{N}=3)$ & $(\mathrm{N}=1)$ & $(\mathrm{N}=1)$ & $(\mathrm{N}=3)$ & $(\mathrm{N}=4)$ & $(\mathrm{N}=1)$ & $(\mathrm{N}=3)$ \\
\hline $\mathrm{Rb}$ & 0.077 & 0.017 & 0.034 & 0.033 & 0.014 & 0.054 & $(<0.010)$ & 0.041 & $(<0.010)$ & 0.035 \\
\hline $\mathrm{Sr}$ & 0.200 & 0.115 & 3.508 & 0.079 & 0.120 & 0.199 & 0.034 & 0.102 & 0.035 & 0.113 \\
\hline Y & 0.104 & 0.111 & 0.089 & 0.025 & 0.057 & 0.040 & 0.037 & 0.065 & 0.074 & 0.062 \\
\hline $\mathrm{Zr}$ & 0.029 & 0.119 & 0.030 & 0.041 & 0.032 & 0.031 & 0.042 & 0.029 & 0.049 & 0.025 \\
\hline $\mathrm{Nb}$ & 0.018 & 0.028 & 0.029 & 0.028 & 0.030 & 0.043 & 0.024 & 0.029 & 0.038 & 0.025 \\
\hline Cs & 0.015 & $(<0.005)$ & 0.011 & $(<0.005)$ & $(<0.005)$ & $(<0.005)$ & $(<0.005)$ & $(<0.005)$ & $(<0.005)$ & 0.006 \\
\hline $\mathrm{La}$ & $(<0.001)$ & 0.003 & 0.001 & 0.002 & $(<0.001)$ & $(<0.001)$ & $(<0.001)$ & $(<0.001)$ & 0.001 & $(<0.001)$ \\
\hline $\mathrm{Ce}$ & $(<0.001)$ & 0.005 & 0.002 & 0.004 & $(<0.001)$ & $(<0.001)$ & 0.002 & $(<0.001)$ & 0.001 & $(<0.001)$ \\
\hline $\operatorname{Pr}$ & $(<0.001)$ & $(<0.001)$ & $(<0.001)$ & $(<0.001)$ & $(<0.001)$ & $(<0.001)$ & $(<0.001)$ & $(<0.001)$ & $(<0.001)$ & $(<0.001)$ \\
\hline $\mathrm{Nd}$ & $(<0.004)$ & $(<0.004)$ & $(<0.004)$ & $(<0.004)$ & $(<0.004)$ & $(<0.004)$ & $(<0.004)$ & $(<0.004)$ & $(<0.003)$ & $(<0.004)$ \\
\hline $\mathrm{Sm}$ & $(<0.006)$ & $(<0.006)$ & $(<0.006)$ & $(<0.006)$ & $(<0.006)$ & $(<0.006)$ & $(<0.006)$ & $(<0.006)$ & $(<0.006)$ & $(<0.006)$ \\
\hline $\mathrm{Eu}$ & $(<0.002)$ & $(<0.002)$ & $(<0.002)$ & $(<0.002)$ & $(<0.002)$ & $(<0.002)$ & $(<0.002)$ & $(<0.002)$ & $(<0.002)$ & $(<0.002)$ \\
\hline $\mathrm{Gd}$ & $(<0.006)$ & $(<0.006)$ & $(<0.006)$ & $(<0.006)$ & $(<0.006)$ & $(<0.006)$ & $(<0.006)$ & $(<0.006)$ & $(<0.006)$ & $(<0.006)$ \\
\hline $\mathrm{Tb}$ & $(<0.002)$ & $(<0.002)$ & $(<0.002)$ & $(<0.002)$ & $(<0.002)$ & $(<0.002)$ & $(<0.002)$ & $(<0.002)$ & $(<0.002)$ & $(<0.002)$ \\
\hline Dy & 0.013 & 0.011 & 0.007 & $(<0.003)$ & $(<0.003)$ & $(<0.003)$ & 0.003 & 0.005 & 0.007 & 0.005 \\
\hline Ho & 0.004 & 0.004 & 0.003 & $(<0.002)$ & $(<0.001)$ & $(<0.002)$ & $(<0.002)$ & 0.003 & 0.003 & 0.002 \\
\hline $\mathrm{Yb}$ & 0.035 & 0.033 & 0.040 & 0.024 & 0.051 & 0.050 & 0.025 & 0.040 & 0.030 & 0.038 \\
\hline $\mathrm{Lu}$ & 0.008 & 0.007 & 0.009 & 0.007 & 0.010 & 0.011 & 0.006 & 0.009 & 0.007 & 0.008 \\
\hline Hf & $(<0.006)$ & $(<0.006)$ & $(<0.006)$ & $(<0.006)$ & $(<0.006)$ & $(<0.006)$ & $(<0.006)$ & $(<0.006)$ & $(<0.006)$ & $(<0.006)$ \\
\hline $\mathrm{Ta}$ & $(<0.002)$ & 0.004 & $(<0.002)$ & $(<0.002)$ & $(<0.002)$ & $(<0.002)$ & $(<0.002)$ & $(<0.002)$ & $(<0.002)$ & $(<0.002)$ \\
\hline $\mathrm{Pb}$ & 0.137 & 0.944 & 0.160 & 0.268 & 0.028 & 0.060 & 0.324 & 0.093 & 0.156 & 0.043 \\
\hline Th & $(<0.003)$ & $(<0.003)$ & $(<0.003)$ & $(<0.003)$ & $(<0.003)$ & $(<0.003)$ & $(<0.003)$ & $(<0.003)$ & $(<0.003)$ & $(<0.004)$ \\
\hline $\mathrm{U}$ & $(<0.003)$ & $(<0.003)$ & $(<0.003)$ & $(<0.003)$ & $(<0.003)$ & $(<0.003)$ & $(<0.003)$ & $(<0.003)$ & $(<0.003)$ & $(<0.003)$ \\
\hline
\end{tabular}

Italic values in parentheses are shown their detection limits when an
(Harz part). Abbreviations of rock lithology are the same as Figure 2. 


\section{Discussion and Summary}

\subsection{Partial Melting Process Recorded in Mantle Harzburgite}

Harzburgite samples from both MTZ and mantle peridotite zones show depleted features, high Fo content of olivine (90-91) and high Cr\# of spinel (0.62-0.77), and are plotted in the trend of OSMA (Figure 7b). The Cr\# of chromian spinel is an indicator of degree of partial melting for residual spinel peridotite or primary melt composition for cumulative ultramafics and volcanic rocks (e.g., [49-51]). It is known to be resistant against alteration and weathering, although changeable via subsolidus formation of Al-rich phase or metasomatism (e.g., [50,52]). In the Mersin's case, there is no obvious formation of subsolidus Al-rich phase around the spinel, e.g., plagioclase (and its alteration product), in both MTZ and mantle harzburgites. There are abundant discordant dunite and pyroxene-rich dikes in the mantle section (cf. Figure 2, Figure 3, and Figure 4e), possibly giving metasomatic effect to the wall-rock harzburgite. However, chromian spinel in the mantle harzburgite samples adjacent to the dunite show no appreciable change in Cr\# (e.g., 2013MR1-02 and 2014MR2-09; 0.72 and 0.73) as compared to other distal harzburgite samples (0.62-0.77) (Table 3). These lines of evidence indicate that the chromian spinel in the Mersin harzburgites has kept its initial chemical features, apart from subsolidus $\mathrm{Mg}-\mathrm{Fe}^{2+}$ exchange, after their partial melting. The Mersin harzburgites are the residual peridotite after a substantially high degree of melting compared to the general abyssal peridotites (e.g., [50]). However, the Fo content of olivine of the Mersin harzburgite shows slightly lower values and some harzburgites are off the partial melting trend of spinel peridotite [40] (Figure 7b). The Fo content of olivine, the $\mathrm{Cr} \#$ of spinel and the HREE contents of clinopyroxene in harzburgite normally show simple depletion trend with increasing the degree of melting (e.g., [50,53]). The Cr\# of spinel shows a simple increase with a decrease of HREE in clinopyroxenes in harzburgites (Figure 13d). Note that the LREE content of clinopyroxene shows no clear correlation with other parameters (Figure 13a,b). These features indicate that partial melting had occurred possibly under the presence of LREE-rich influx. No negative correlation between HREE content of clinopyroxene and Fo content of olivine (Figure 13c) implies the addition of Fe during partial melting. In addition, high concentration of some LILE (e.g., $\mathrm{Sr}$ and $\mathrm{Pb}$ ) are possibly resulted from the addition of such elements during the partial melting (Table 6). Aqueous fluid, which is rich in LREE, LILE, and Fe, is a candidate as the influx, and high Cr\# of spinel in the Mersin harzburgite (up to 0.8) is consistent with the high-degree partial melting under hydrous condition (e.g., [54]). Then, the harzburgite of the Mersin ophiolite is possibly residue after high-degree partial melting assisted by influx of aqueous fluid(s) or hydrous melt(s), which were rich in Fe, LILE, and LREE.

To estimate the degree of partial melting of residual mantle peridotite, HREE concentrations in clinopyroxene are quite useful (e.g., [53,55]) because they are relatively unchangeable during metasomatism and weathering, although they could be slightly lowered by formation of subsolidus clinopyroxenes and subsolidus trace-element redistribution with other peridotitic minerals (e.g., [56,57]). Here we do not estimate the subsolidus effects for REE in clinopyroxene and orthopyroxene, because such subsolidus redistribution effect for HREE is relatively small. We roughly estimated the degree of melting by using HREE compositions of clinopyroxene for both the MTZ and mantle harzburgites, to be approximately 25\% melting of depleted MORB mantle (=DMM; [58]) at spinel stability field. We also checked the possibility of melting at garnet stability field. The calculated melting degree of DMM at the garnet stability field is mostly 5\% followed by $18-20 \%$ melting of the residue at the spinel stability field. The HREE concentrations of orthopyroxene are also a good measure for the partial melting event (e.g., [59]). Calculated degree of melting of DMM by using HREE composition of orthopyroxene is $10 \%$ at garnet stability field and $10 \%$ at spinel stability field. These values are consistent with the high Cr\# (up to 0.8) of chromian spinel in the Mersin harzburgite, which could be attained by a relatively high degree (> 20\%) of melting of peridotite with an influx of $\mathrm{H}_{2} \mathrm{O}$-bearing fluid or melt (e.g., [54,60]). 

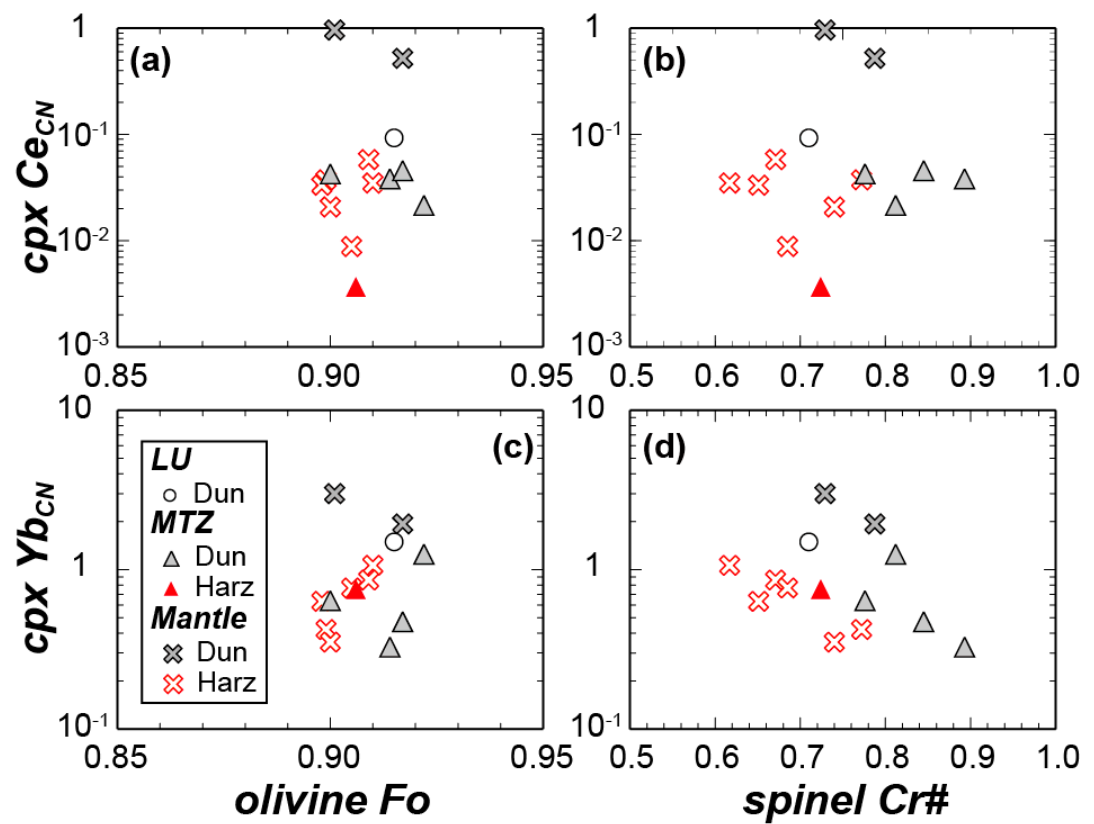

Figure 13. Relationships between major-element mineral (olivine and chromian spinel) compositions and $\mathrm{REE}_{\mathrm{CN}}$ in coexisting clinopyroxene in dunite and residual harzburgite. (a) The Fo content of olivine and $\mathrm{Ce}_{\mathrm{CN}}$ of clinopyroxene. (b) The $\mathrm{Cr} \#$ of chromian spinel and $\mathrm{Ce}_{\mathrm{CN}}$ of clinopyroxene. (c) The Fo content of olivine and $\mathrm{Yb}_{\mathrm{CN}}$ of clinopyroxene. (d) The Cr\# of chromian spinel and $\mathrm{Yb}_{\mathrm{CN}}$ of clinopyroxene.

\subsection{Metasomatism by $\mathrm{SiO}_{2}$-Rich Fluid/Melt Suggested by High-Ni Olivine of Harzburgite}

Some olivines in harzburgites both from MTZ and from the mantle section show obviously high $\mathrm{NiO}$ content off the "mantle olivine array" (Figure 7a). To increase $\mathrm{NiO}$ content of olivine, Ni addition is needed and could be supplied from surrounding olivine, replaced by secondary orthopyroxene (cf. Figure 6d) due to infiltration of $\mathrm{SiO}_{2}$-rich melt or fluid: $2(\mathrm{Mg}, \mathrm{Fe}, \mathrm{Ni})_{2} \mathrm{SiO}_{4}$ (olivine) $+\mathrm{SiO}_{2}$ (melt/fluid) $=2(\mathrm{Mg}, \mathrm{Fe}) \mathrm{SiO}_{3}$ (orthopyroxene) $+\mathrm{Ni}_{2} \mathrm{SiO}_{4}$ (Ni-rich olivine component) [61]. This is due to a $>$ two times higher distribution coefficients of $\mathrm{Ni}$ between silicate melt and olivine than those between silicate melt and orthopyroxene [62]. However, local Ni enrichment has not been observed even on olivine grains adjacent to any orthopyroxene grains in individual samples. These imply that such a metasomatic event was relatively old and the local metasomatic Ni enrichment in olivine obscured by subsequent partial melting of the metasomatized peridotite (Figure 14). The subsequent partial melting of the metasomatized peridotite consumed both orthopyroxenes, i.e., primary and secondary, and left no obvious texture of orthopyroxene enrichment in the Mersin harzburgite (cf. Figure 5), which is in contrast to the clear replacement textures of secondary orthopyroxene formed by young metasomatism in sub-arc peridotite xenoliths (e.g., [1]).

\subsection{Nature and Formation Process of Dunites, Wehrlite and Pyroxenites}

As for the origin of dunite, we have three possibilities: i.e., (1) Residual dunite after extremely high degree of partial melting of peridotite (residual origin); (2) a cumulus dunite crystallized from primitive mantle melt; and (3) a replacive dunite formed by interaction between residual mantle harzburgite and upwelling mantle melt. If the dunite is of residual origin, it is expected to occur at the uppermost mantle and to show extremely depleted mineral chemistry, i.e., high Cr\# of spinel, high Fo content of olivine (e.g., [63]) and low incompatible trace element concentrations of clinopyroxene (e.g., [64]). Clinopyroxenes in LU dunites show slightly higher REE abundances than in the both harzburgites of MTZ and mantle section (Figure 11). In addition, the LU dunite shows adcumulate and mesocumulate textures [29] and the mineral chemistry, e.g., abrupt decrease of $\mathrm{NiO}$ content of olivine 
in harmony with Fo content of olivine (Figure 7a), also supports its cumulus origin. On the other hand, the MTZ dunite often contains olivine grains showing wavy extinction and kink bands (Figure 6b) and do not show obvious cumulus textures and depleted chemical features of minerals, e.g., high Cr\# of chromian spinel (Figure 8a) and low HREE abundance of clinopyroxene than mantle residual peridotite (Figures $11 \mathrm{~b}$ and 13). However, abrupt decrease of $\mathrm{NiO}$ content of olivine with decreasing Fo content of olivine implies their fractional crystalized character (Figure $7 \mathrm{~b}$ ). These features deny its simple cumulus nature. A simple residual origin is not accepted either for the MTZ dunite because of the presence of modal gap between dunite and harzburgites (Figure 5) [65]. Nicolas and Prinzhofer [66] discussed the petrogenesis of MTZ dunite and mentioned possible replacive nature of most of the MTZ dunite in the Oman ophiolite, and the Mersin MTZ dunite is possibly of such replacive origin. The presence of chromitite in MTZ dunite of the Mersin ophiolite also supports their replacive origin based on the idea of petrogenesis of chromitite, which is a reaction product of mantle peridotite and relatively primitive basaltic magmas (e.g., [67]).

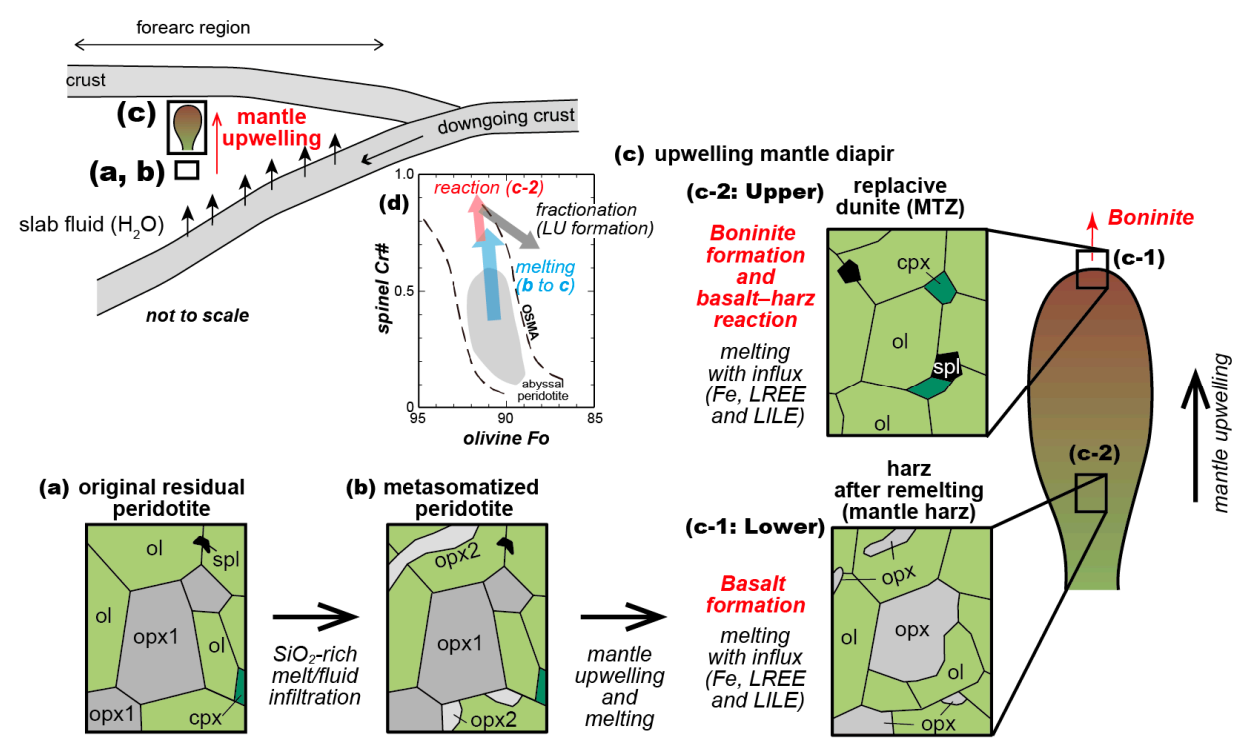

Figure 14. Schematic model showing evolution of the Mersin ophiolite. We suggest that the Mersin ophiolite was formed at a frontal part of suprasubduction zone setting. Abbreviations of minerals are the same in Figure 6. (a) Original peridotite experienced partial melting with assistance of slab-derived aqueous fluid, which was rich in LREE, LILE and Fe at relatively deeper part of mantle. (b) The original peridotite had been metasomatized by $\mathrm{SiO}_{2}$-rich melt and/or fluid to form secondary orthopyroxene at expense of olivine prior to partial melting event. (c) Buoyant Mg-rich metasomatized mantle with some amount of low-density partial melt started to ascend and partially melted. (c-1) In the deeper part of the upwelling diapir, the metasomatized peridotite was remelted to produce high-Mg basaltic to boninitic melt and highly depleted residual mantle harzburgite now observed in the Mersin mantle section. (c-2) In the upper part of the upwelling diapir, such Mg-rich basaltic and boninitic melt, was accumulated at the top and reacted with the roof harzburgite to produce boninite and left highly depleted dunite (MTZ rocks). The ascending boninitic melt formed cumulus LU upsection. (d) Schematic image of chemical evolution during these stages. Cr\# of spinel in the Mersin ultramafic rocks has increased during partial melting and reaction stage, i.e., from panel (b) to (c), and in the fractionation stage, cumulus LU has formed from ascending boninitic melt.

The calculated melt in equilibrium with clinopyroxene in the LU rocks, including clinopyroxenite and wehrlite, shows lower REE abundances than the Mersin effusive rocks (tholeiitic basalt), although similar in REE pattern and concentrations to boninite, except for the slightly lower LREE abundance for the former (Figure 11b). Boninites from Izu-Ogasawara arc show variable LREE concentrations, which were interpreted to be due to the difference in amount of added slab component [48]. The high 
LREE may indicate high contributions of slab component [68]. Mineral chemistry and mineral assemblages of LU rocks, which are high-Mg\# of olivine and clinopyroxene and free of plagioclase, suggests their high-pressure crystallization ( $\approx 1$ GPa: [29,69]).

The melt responsible for formation of the high-Mg MTZ dunite, which contains high-Cr\# spinel (Figures 7b and 8), was likely in-situ produced at the level of MTZ. No sign for the production or passage of such melt has been left in the mantle section (Figure 7). To produce a replacive MTZ dunite with a highly depleted nature (higher Cr\# of chromian spinel, Fo content of olivine and lower HREE clinopyroxene), we need a highly depleted reaction system, where both or either of wall harzburgite and infiltrated melt should be depleted. The calculated melt in equilibrium with clinopyroxene in the MTZ dunite shows a similar REE pattern with boninite, although varied in LREE MREE content (Figure 11b). This variation of REE patterns of clinopyroxene in MTZ dunite possibly indicates a variation of melt volume to the wall rock harzburgite, and the MREE-depleted spoon-shaped pattern of the calculated melt implies that the clinopyroxene had not achieved equilibration in terms of REE content with the infiltrated melt (Figure 11b). The higher Fo content of the dunite olivine in MTZ than in any harzburgite of the Mersin ophiolite implies predominance of the factor of melting of already depleted harzburgite during the interaction with infiltrated melt. Such a phenomenon possibly occurs around the top of upwelling mantle diapir of depleted peridotite, which is relatively buoyant because of the higher-Mg feature with possible presence of low-density partial melt(s) [70-72]. The partial melting produced high-Mg basalt to boninitic melt, possibly assisted by slab-derived hydrous components. The secondary addition of metasomatic orthopyroxene may have contributed to enhance the volume of the partial melt accumulated at the diapir top (Figure 14). Such basaltic to boninitic melt actively reacted with the roof harzburgite overlying the diapir to produce boninite and highly depleted dunite [73]. Additionally, the ascending melt formed cumulus LU rocks upsection. The MTZ wehrlite possibly represents the melt-rich portion of the reaction zone because REE abundances are high both in clinopyroxene and the calculated equilibrium melt. The melt moved upward to formed the LU rocks upsection because the REE pattern of clinopyroxene is almost the same between the MTZ wehrlite and the LU rocks (Figure 11).

Olivines in the mantle dunite does not show obviously low $\mathrm{NiO}$ content and low Fo content as observed in LU and MTZ dunites, and are plotted in the residual mantle peridotite field (Figure 7). However, the mantle dunite crosscuts the deformation structure of the mantle harzburgite (Figure 4e) and is of replacive origin (e.g., [74]). The higher $\mathrm{TiO}_{2}$ content of chromian spinel in the mantle dunite (Figure $8 b$ ) and the higher REE levels of the clinopyroxenes in the mantle dunite also support its replacive origin. The calculated melt in equilibrium with clinopyroxene in mantle dunite shows almost the same pattern as that of MTZ dunite, although the abundance is higher and approaches to that of the Mersin tholeiitic basalt (Figure 11b). These characters indicate the infiltrated melt was possibly formed at much deeper part of the mantle, and the mantle dunite could be a fossil of melt conduit to feed the Mersin tholeiitic basalt upward. The variation of REE patterns of clinopyroxene in mantle dunite possibly reflects variable volume ratios of the melt to the wall rock harzburgite, and the melt volume was maybe low based on relatively high $\mathrm{NiO}$ content of olivine (Figure 7a) in respect of high partition coefficient of $\mathrm{Ni}$ in olivine relative to melt (e.g., [62]). If the melt volume was high, the $\mathrm{NiO}$ content of olivine decreased abruptly as observed in LU dunite (Figure 7a). The MREE-depleted spoon-shaped pattern of the calculated melt in equilibrium with those clinopyroxene implies incomplete equilibration with the infiltrated melt in terms of REE, as observed in the MTZ dunite (Figure 11).

\subsection{Implications for Tectonic Setting and History of the Mersin Ophiolite}

The geochemical features of the Mersin ultramafic rocks show their similarity to the present arc-related rocks rather than MORB-related ones (Figure 8), confirming the arc affinity of the Mersin ophiolite, except for the old (Late Jurassic to Early Cretaceous) alkaline basalt [9]. The whole section of the Mersin ophiolite was formed at a suprasubduction zone condition. In addition, the boninite-related features of MTZ and lower crustal section (LU section) specify that they were formed at this level. 
It is noteworthy that the MTZ is thicker $(>1 \mathrm{~km}$ ) than in the Oman ophiolite (mostly $<500 \mathrm{~m}$ in thickness) [75], which recorded the switch of tectonic setting from a mid-ocean ridge to an arc [4,5].

Residual harzburgite shows highly depleted character, i.e., high Cr\# of chromian spinel (0.6-0.8) (Figure $7 b$ ) and estimated high degree of partial melting (> 20\%). In addition, it records $\mathrm{Si}$ metasomatism, which formed olivine-replacing orthopyroxene (Figure 6d) and high $\mathrm{NiO}$ content of remaining olivine (Figure 7a). Presence of such metasomatic orthopyroxene has been reported in peridotite xenoliths from several localities of arc setting (e.g., [1]), and is interpreted as reaction product between the peridotite and $\mathrm{SiO}_{2}$-rich melt or aqueous fluid derived from subducting slab. We conclude that this is one of the representative features in arc mantle. However, the lack of obvious correlation between modal amount of orthopyroxene and $\mathrm{NiO}$ content of olivine suggests that the formation of the secondary orthopyroxene is relatively old prior to partial melting, which obscured modal proportion of olivine/orthopyroxene and replacement texture (Figure 14).

The partial melting was due to upwelling of Mg-rich and melt-retaining buoyant peridotite enriched with orthopyroxene and was also assisted by LREE-, LILE-, and Fe-rich slab-derived fluid. The melting of the upwelling diapir produced Mg-rich basaltic-boninitic melts (Figure 14). The basic geochemical features of the residual mantle harzburgite of the Mersin ophiolite were obtained in this stage, and some modifications have occurred only around the discordant dunite or pyroxenite dikes. The produced melt was accumulated at the top, where the degree of partial melting is highest, and interaction with the roof harzburgite occurred to produce boninite and dunitic peridotites (Figure 14). The MTZ is representative of the reaction zone. The boninitic melt formed by the reaction migrated to shallower part and formed cumulus LU rocks. The ultramafic rocks cumulated from the LU rocks downsection of the Mersin ophiolite are free of plagioclase [29], which indicates the formation of such boninitic magma at a pressure higher than $1 \mathrm{GPa}$.

After the formation of the Mersin ophiolite sequence, i.e., from residual mantle to ultramafic-mafic cumulates, the production of arc-type lavas (IAT tholeiite) has been a main volcanic event and such tholeiitic magmas formed discordant dunite (mantle dunite) in the mantle harzburgite (harzburgite in mantle section). Many diabase dikes with tholeiitic affinity have been observed in all levels of the ophiolite, including metamorphic sole [10], suggesting the continuous IAT tholeiite production until complete the ophiolite obduction.

Author Contributions: S.I. and S.A. conceived this contribution and mainly wrote the manuscript assisted by all other co-authors; S.I., Y.S., M.M., O.P. and S.A. performed the field investigation and sample collection; Y.S. and M.M. contributed sample preparation (making thin sections) for observation, EPMA and LA-ICPMS analyses; Y.S. and S.I. performed the EPMA and LA-ICPMS analyses; S.I., Y.S. and S.A. analyzed the data.

Funding: This research was partly supported by MONKASHO SPECIAL BUDGET “Decoding ocean-floor dynamics from ophiolites" to S.A., Japan Society for the Promotion of Science KAKENHI Grant Numbers JP24540518: (Grant-in-Aid for Scientific Research (C)) and JP16K1783400 (Grant-in-Aid for Young Scientists (B)) to S.I. and Open Fund (GPMR201702) of State Key Lab. of Geological Processes and Mineral Resources, China University of Geosciences, Wuhan, to O.P.

Acknowledgments: We greatly appreciate the assistance of T. Yamamuro, T. Nishiyama and H. Isobe during microprobe analyses at Kumamoto University. Tomoaki Morishita and Akihiro Tamura supported EPMA and LA-ICPMS analyses done by Yuji Saikawa at Kanazawa University. Norikatsu Akizawa helped us sampling during filed work in 2013. Comments from two anonymous reviewers improved the manuscript.

Conflicts of Interest: The authors declare no conflict of interest.

\section{References}

1. Arai, S.; Ishimaru, S. Insights into petrological characteristics of the lithosphere of mantle wedge beneath arcs through peridotite xenoliths: A review. J. Petrol. 2008, 49, 665-695. [CrossRef]

2. Lippard, S.J.; Shelton, A.W.; Gass, I.G. The Ophiolite of Northern Oman. Geol. Soc. Lond. Mem. 1986, 11, 178.

3. Dilek, Y.; Furnes, H. Ophiolite genesis and global tectonics: Geochemical and tectonic fingerprinting of ancient oceanic lithosphere. Geol. Soc. Am. Bull. 2011, 123, 387-411. [CrossRef] 
4. Boudier, F.; Nicolas, A.; Bouchez, J.L. Kinematics of oceanic thrusting and subduction from basal sections of ophiolites. Nature 1982, 296, 825-828. [CrossRef]

5. Arai, S.; Kadoshima, K.; Morishita, T. Widespread arc-related melting in the mantle section of the northern Oman ophiolite as inferred from detrital chromian spinels. J. Geol. Soc. Lond. 2006, 163, 869-879. [CrossRef]

6. Uysal, I.; Ersoy, E.Y.; Karslı, O.; Dilek, Y.; Sadıklar, M.B.; Ottley, C.J.; Tiepolo, M.; Meisel, T. Coexistence of abyssal and ultra-depleted SSZ type mantle peridotites in a Neo-Tethyan Ophiolite in SW Turkey: Constraints from mineral composition, whole-rock geochemistry (major-trace-REE-PGE), and Re-Os isotope systematics. Lithos 2012, 132-133, 50-69. [CrossRef]

7. Kay, S.M.; Kay, R.W. Role of crystal cumulates and the oceanic crust in the formation of the lower crust of the Aleutian arc. Geology 1985, 13, 461-464. [CrossRef]

8. Parlak, O. The Tauride ophiolites of Anatolia (Turkey): A review. J. Earth Sci. 2016, 27, 901-934. [CrossRef]

9. Parlak, O.; Delaloye, M.; Bíngöl, F. Geochemistry of the volcanic rocks in the Mersin ophiolite (Southern Turkey) and their tectonic significance in the Eastern Mediterranean geology. In Proceedings of the International Earth Sciences Colloquium on the Aegean Region (IESCA-95), Izmir-Güllük, Turkey, 9-14 October 1995; Piskin, Ö., Ergün, M., Savaşçın, M.Y., Tarcan, G., Eds.; Dokuz Eylul University: İzmir, Turkey, 1997; pp. 441-463.

10. Parlak, O.; Delaloye, M. Geochemistry and timing of post-metamorphic dyke emplacement in the Mersin Ophiolite (southern Turkey): New age constraints from ${ }^{40} \mathrm{Ar} /{ }^{39} \mathrm{Ar}$ geochronology. Terra Nova 1996, 8, 585-592. [CrossRef]

11. Ketin, I. Tectonic units of Anatolia. MTA Bull. 1966, 66, 23-24.

12. Şengör, A.M.C.; Yılmaz, Y. Tethyan Evolution of Turkey: A Plate Tectonic Approach. Tectonophysics 1981, 75, 181-241. [CrossRef]

13. Robertson, A.H.F.; Dixon, J.A. Introduction: Aspects of the Geological Evolution of the Eastern Mediterranean. In The Geological Evolution of the Eastern Mediterranean; Dixon, J.A., Robertson, A.H.F., Eds.; Geological Society of London: London, UK, 1984; Volume 17, pp. 1-74.

14. Görür, N.; Oktay, F.Y.; Seymen, İ.; Şengör, A.M.C. Palaeotectonic Evolution of the Tuzgölü Basin Complex, Central Turkey: Sedimentary Record of a Neotethyan Closure. In The Geological Evolution of the Eastern Mediterranean; Dixon, J.A., Robertson, A.H.F., Eds.; Geological Society of London: London, UK, 1984; Volume 17 , pp. 467-482.

15. Okay, A.İ. High-Pressure/Low-Temperature Metamorphic Rocks of Turkey. In Blueschists and Eclogites; Evans, B.W., Brown, E.H., Eds.; Geological Society of America: Boulder, CO, USA, 1986; Volume 164, pp. 333-348.

16. Yilmaz, Y. New evidence and model on the evolution of the southeast Anatolian Orogen. Geol. Soc. Am. Bull. 1993, 105, 251-271. [CrossRef]

17. Yılmaz, Y.; Yiğitbaş, E.; Genç, Ş.C. Ophiolitic and metamorphic assemblages of southeast Anatolia and their significance in the geological evolution of the orogenic belt. Tectonics 1993, 12, 1280-1297. [CrossRef]

18. Okay, A.İ.; Tüysüz, O. Tethyan Sutures of Northern Turkey. In The Mediterranean Basins: Tertiary Extension within the Alpine Orogeny; Mascle, A., Jolivet, L., Eds.; Geological Society of London: London, UK, 1999; Volume 156, pp. 475-516.

19. Robertson, A.H.F. Overview of the genesis and emplacement of Mesozoic ophiolites in the Eastern Meditterranean Tethyan region. Lithos 2002, 65, 1-67. [CrossRef]

20. Stampfli, G.M.; Borel, G.D. A plate tectonic model for the Palaeozoic and Mesozoic constrained by dynamic plate boundaries and restored synthetic oceanic isochrones. Earth Planet. Sci. Lett. 2002, 169, 17-33. [CrossRef]

21. Robertson, A.H.F.; Ustaomer, T. Tectonic evolution of the Intra-Pontide suture in the Armautlu Peninsula, NW Turkey. Tectonophysics 2004, 381, 175-209. [CrossRef]

22. Oberhansli, R.; Candan, O.; Bousquet, R.; Rimmele, G.; Okay, A.; Goff, J.B. Alpine HP evolution of the eastern Bitlis complex, SE Turkey. In Sedimentary Basin Tectonics from the Black Sea and Caucasus to the Arabian Sea; Sosson, M., Kaymakci, N., Stephenson, R., Bergerat, F., Starostenko, V., Eds.; Geological Society of London: London, UK, 2010; Volume 340, pp. 461-483.

23. Pourteau, A.; Candan, O.; Oberhänsli, R. High-Pressure metasediments in Central Turkey: Constraints on the Neotethyan Closure History. Tectonics 2010, 29, TC5004. [CrossRef] 
24. Robertson, A.H.F.; Parlak, O.; Ustaömer, T. Overview of the Palaeozoic-Neogene Evolution of Neotethys in the Eastern Mediterranean Region (Southern Turkey, Cyprus, Syria). Petrol. Geosci. 2012, 18, 381-404. [CrossRef]

25. Robertson, A.H.F.; Parlak, O.; Ustaömer, T. Late-Palaeozoic-Early Cenozoic tectonic development of Southern Turkey and the easternmost Mediterranean region: Evidence from the inter-relations of continental and oceanic units. In Geological Development of Anatolia and the Easternmost Mediterranean Region; Robertson, A.H.F., Parlak, O., Ünlügenç, U.C., Eds.; Geological Society of London: London, UK, 2013; Volume 372, pp. 9-48.

26. Andrew, T.; Robertson, A.H.F. The Beyşehir-Hoyran-Hadim Nappes: Genesis and Emplacement of Mesozoic Marginal and Oceanic Units of the Northern Neotethys in Southern Turkey. J. Geol. Soc. 2002, 159, 529-543. [CrossRef]

27. Parlak, O.; Robertson, A.H.F. The ophiolite-related Mersin mélange, southern Turkey: Its role in the tectonic-Sedimentary setting of Tethys in the Eastern Mediterranean region. Geol. Mag. 2004, 141, 257-286. [CrossRef]

28. Robertson, A.H.F. Development of Concepts Concerning the Genesis and Emplacement of Tethyan Ophiolites in the Eastern Mediterranean and Oman Regions. Earth Sci. Rev. 2004, 66, 331-387. [CrossRef]

29. Parlak, O.; Delaloye, M.; Bíngöl, F. Mineral chemistry of ultramafic and mafic cumulates as an indicator of the arc-related origin of the Mersín ophiolite (southern Turkey). Geol. Rund. 1996, 85, 647-661. [CrossRef]

30. Parlak, O.; Delaloye, M.; Bíngöl, F. Phase and cryptic variation through the ultramafic-mafic cumulates in the Mersin ophiolite (southern Turkey). Ofioliti 1996, 21, 81-92.

31. Boudier, F.; Nicolas, A. Nature of the Moho transition xone in the Oman ophiolite. J. Petrol. 1995, 36, 777-796. [CrossRef]

32. Parlak, O.; Delaloye, M.; Bíngöl, F. Origin of sub-ophiolitic metamorphic rocks beneath the Mersin ophiolite, southern Turkey. Ofioliti 1995, 20, 97-110.

33. Çelik, Ö.F. Detailed Geochemistry and K-Ar Geochronology of the Metamorphic Sole Rocks and Their Mafic Dykes from the Mersin Ophiolite, Southern Turkey. Turk. J. Earth Sci. 2008, 17, 685-708.

34. Dilek, Y.; Thy, P.; Hacker, B.; Grundvig, S. Structure and petrology of Tauride ophiolites and mafic dike intrusions (Turkey): Implications for the Neotethyan ocean. Geol. Soc. Am. Bull. 1999, 111, 1192-1216. [CrossRef]

35. Arai, S. Igneous mineral equilibria in some Alpine-type peridotites in Japan. In Materials Science in Earth's Interior; Sunagawa, I., Ed.; Terra: Tokyo, Japan, 1984; pp. 445-460.

36. Morishita, T.; Ishida, Y.; Arai, S.; Shirasaka, M. Determinaion of meltiple trace element compositions in thin $(<30 \mu \mathrm{m})$ lasers of NIST SRM 614 and 616 using laser albration-inductively coupled plasma-mass spectrometry (LA-ICP-MS). Geostand. Geoanal. Res. 2005, 29, 107-122. [CrossRef]

37. Leake, B.E.; Woolley, A.R.; Arps, C.E.S.; Birch, W.D.; Gilbert, M.C.; Grice, J.D.; Hawthorne, F.C.; Kato, A.; Kisch, H.J.; Krivovichev, V.G.; et al. Nomenclature of amphiboles report of the subcommittee on amphiboles of the international mineralogical association commission on new minerals and mineral names. Eur. J. Miner. 1997, 9, 623-651. [CrossRef]

38. Takahashi, E. Origin of basaltic magmas: Implications from peridotite melting experiments and an olivine fractionation model. Bull. Vol. Soc. Jpn. 1986, 30, S17-S40, (In Japanese with English Abstract).

39. Herzburg, C.; Asimow, P.D.; Ionov, D.A.; Vidito, C.; Jackson, M.G.; Geist, D. Nickel and helium evidence for melt above the core-mantle boundary. Nature 2013, 494, 393-398. [CrossRef] [PubMed]

40. Arai, S. Origin of ophiolitic peridotites. J. Geogr. 1989, 3, 45-54, (In Japanese with English abstract). [CrossRef]

41. Tamura, A.; Arai, S. Harzburgite-dunite-orthopyroxenite suite as a record of supra-subduction zone setting for the Oman ophiolite mantle. Lithos 2006, 90, 43-56. [CrossRef]

42. Arai, S.; Okamura, H.; Kadoshima, K.; Tanaka, C.; Suzuki, K.; Ishimaru, S. Chemical characteristics of chromian spinel in plutonic rocks: Implications for deep magma processes and discrimination of tectonic setting. Island Arc 2011, 20, 125-137. [CrossRef]

43. Arai, S. Dunite-harzburgite-chromitite complexes as refractory residue in the Sangun-Yamaguchi zone, western Japan. J. Petrol. 1980, 21, 141-165. [CrossRef]

44. McDonough, W.F.; Sun, S.-S. The composition of the Earth. Chem. Geol. 1995, 120, 223-253. [CrossRef]

45. Johnson, K.T.M.; Dick, H.J.B. Open system melting and temporal and spatial variation of peridotite and basal at the Atlantis II fracture zone. J. Geophys. Res. 1992, 97, 9219-9241. [CrossRef] 
46. Workman, R.K.; Hart, S.R. Major and trace element composition of the depleted MORB mantle (DMM). Earth Planet. Sci. Lett. 2005, 231, 53-72.

47. Warren, J.M.; Shimizu, N. Cryptic variations in abyssal peridotite compositions: Evidence for shallow-level melt infiltration in the oceanic lithosphere. J. Petrol. 2010, 51, 395-423. [CrossRef]

48. Kanayama, K.; Umino, S.; Ishizuka, O. Eocene volcanism during the incipient stage of Izu-Ogasawara Arc: Geology and petrology of the Mukojima Island Group, the Ogasawara Islands. Island Arc 2012, 21, 288-316. [CrossRef]

49. Dick, H.J.B.; Bullen, T. Chromian spinel as a petrogenetic indicator in abyssal and alpine-type peridotites as spatially associated lavas. Contrib. Miner. Petrol. 1984, 86, 54-76. [CrossRef]

50. Arai, S. Characterization of spinel peridotites by olivine-spinel compositional relationships: Review and interpretation. Chem. Geol. 1994, 113, 191-204. [CrossRef]

51. Arai, S. Compositional variation of olivine-chrominal spinel in $\mathrm{Mg}$-rich magmas as a guide to their residual spinel peridotites. J. Volcanol. Geoth. Res. 1994, 59, 279-293. [CrossRef]

52. Rampone, R.; Piccardo, G.B.; Vannucci, R.; Bottazzi, P.; Ottolini. Subsolidus reactions monitored by traceelement partitioning: The spinel- to plagioclase-facies transition in mantle peridotites. Contrib. Miner. Petrol. 1993, 115, 1-17. [CrossRef]

53. Hellebrand, E.; Snow, J.E.; Dick, H.J.; Hofmann, A.W. Coupled major and trace elements as indicators of the extent of melting in mid-ocean-ridge peridotites. Nature 2001, 410, 677-681. [CrossRef] [PubMed]

54. Matsukage, K.N.; Kubo, K. Chromian spinel during melting experiments of dry peridotite (KLB-1) at 1.0-2.5 GPa. Am. Miner. 2003, 88, 1271-1278. [CrossRef]

55. Johnson, K.T.M.; Dick, H.J.B.; Shimizu, N. Melting in the oceanic upper mantle: An ion microprobe study of diopsides in abyssal peridotites. J. Geophys. Res. 1990, 95, 2661-2678. [CrossRef]

56. Witt-Eickschen, G.; O'Neill, H.S. The effect of temperature on the equilibrium distribution of trace elements between clinopyroxene, orthopyroxene, olivine and spinel in upper mantle peridotite. Chem. Geol. 2005, 221, 65-101. [CrossRef]

57. Sun, C.; Liang, Y. An assessment of subsolidus re-equilibration on REE distribution among mantle minerals olivine, orthopyroxene, clinopyroxene and garnet in peridotite. Chem. Geol. 2014, 372, 80-91. [CrossRef]

58. Salters, V.J.M.; Stracke, A. Composition of the depleted mantle. Geochem. Geophys. Geosyst. 2004, 5, Q05004. [CrossRef]

59. Scott, J.M.; Liu, J.; Pearson, D.G.; Waight, T.E. Mantle depletion and metasomatism recorded in orthopyroxene in highly depleted peridotites. Chem. Geol. 2016, 441, 280-291. [CrossRef]

60. Hirose, K.; Kawamoto, T. Hydrous partial melting of lherzolite at $1 \mathrm{GPa}$ : The effect of $\mathrm{H}_{2} \mathrm{O}$ on the genesis of basaltic magmas. Earth Planet. Sci. Lett. 1995, 133, 463-473. [CrossRef]

61. Kelemen, P.B.; Hart, S.R.; Bernstein, S. Silica enrichment in the continental upper mantle via melt/rock reaction. Earth Planet. Sci. Lett. 1998, 164, 387-406. [CrossRef]

62. Straub, S.M.; LaGatta, A.B.; Martin-Del Pozzo, A.L.; Langmuir, C.H. Evidence from high-Ni olivines for a hybridized peridotite/pyroxenite source for orogenic andesites from the central Mexican Volcanic Belt. Geochem. Geophys. Geosyst. 2008, 9, Q03007. [CrossRef]

63. Mysen, B.; Kushiro, I. Compositional variations of coexisting phases with degree of melting of peridotite in the upper mantle. Am. Miner. 1977, 62, 843-865.

64. Prinzhofer, A.; Allégre, C.J. Residual peridotites and the mechanisms of partial melting. Earth Planet. Sci. Lett. 1985, 74, 251-265. [CrossRef]

65. Presnall, D.C. The geometrical analysis of partial fusion. Am. J. Sci. 1969, 267, 1178-1194. [CrossRef]

66. Nicolas, A.; Prinzhofer, A. Cumulative or residual origin for the transition zone in ophiolites: Structural evidence. J. Petrol. 1983, 24, 188-206. [CrossRef]

67. Arai, S.; Yurimoto, H. Podiform chromitites of the Tari-Misaka ultramafic complex, southwestern Japan, as mantle-melt interaction products. Econ. Geol. 1994, 89, 1279-1288. [CrossRef]

68. Kessel, R.; Shmidt, M.W.; Ulmer, P.; Pettke, T. Trace element signature of subduction-zone fluids, melts and supercritical liquids at 120-180 km depth. Nature 2005, 437, 724-727. [CrossRef] [PubMed]

69. Elthon, D.; Casey, J.F.; Komor, S. Mineral chemistry of ultramafic cumulates from the North Arm Mountain massif of the Bay of Islands ophiolite: Evidence for high-pressure crystal fractionation of oceanic basalt. J. Geophys. Res. 1982, 87, 8717-8734. [CrossRef] 
70. Barnouin-Jha, K.; Parmentier, E.M.; Sparks, D.W. Buoyant mantle upwelling and crustal production at oceanic spreading centers: On-axis segmentation and off-axis melting. J. Geophys. Res. 1997, 102, 11979-11989. [CrossRef]

71. Hernlund, J.W.; Stevenson, D.J.; Tackley, P.J. Buoyant melting instabilities beneath extending lithosphere: 1. Numerical models. J. Geophys. Res. 2008, 113, B04405. [CrossRef]

72. Hernlund, J.W.; Stevenson, D.J.; Tackley, P.J. Buoyant melting instabilities beneath extending lithosphere: 2. Linear analysis. J. Geophys. Res. 2008, 113, B04406. [CrossRef]

73. Fisk, M.R. Basalt magma interaction with harzburgite and the formation of high-magnesian andesites. Geophys. Res. Lett. 1986, 13, 467-470. [CrossRef]

74. Kelemen, P.B. Reaction between ultramafic rocks and fractionating basaltic magma. I. Phase relations, the origin of calc-alkaline magma series, and the formation of discordant dunite. J. Petrol. 1990, 31, 51-98. [CrossRef]

75. Nicolas, A.; Boudier, F.; Ildefonse, B. Variable crustal thickness in the Oman ophiolite: Implication for oceanic crust. J. Geophys. Res. 1996, 191, 17941-17950. [CrossRef]

(C) 2018 by the authors. Licensee MDPI, Basel, Switzerland. This article is an open access article distributed under the terms and conditions of the Creative Commons Attribution (CC BY) license (http://creativecommons.org/licenses/by/4.0/). 